\title{
CONSTRAINTS ON COSMIC RAYS, MAGNETIC FIELDS, AND DARK MATTER FROM GAMMA-RAY OBSERVATIONS OF THE COMA CLUSTER OF GALAXIES WITH VERITAS AND FERMI
}

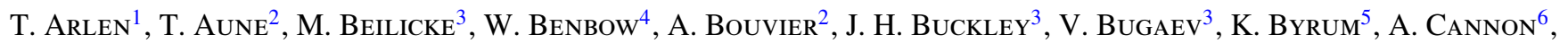
A. Cesarini ${ }^{7}$, L. CiupiK ${ }^{8}$, E. Collins-Hughes ${ }^{6}$, M. P. Connolly ${ }^{7}$, W. Cui ${ }^{9}$, R. Dickherber ${ }^{3}$, J. Dumm ${ }^{10}$, A. FalCone ${ }^{11}$, S. Federici ${ }^{12,13}$, Q. Feng ${ }^{9}$, J. P. Finley ${ }^{9}$, G. Finnegan ${ }^{14}$, L. Fortson ${ }^{10}$, A. Furniss ${ }^{2}$, N. Galante ${ }^{4}$, D. Gall ${ }^{15}$, S. Godambe ${ }^{14}$, S. Griffin ${ }^{16}$, J. Grube ${ }^{8}$, G. GyuK ${ }^{8}$, J. Holder ${ }^{17}$, H. Huan ${ }^{18}$, G. Hughes ${ }^{12}$, T. B. HumenskY ${ }^{19}$, A. Imran ${ }^{20}$, P. KaAReT ${ }^{15}$, N. Karlsson ${ }^{10}$, M. Kertzman ${ }^{21}$, Y. Khassen ${ }^{6}$, D. Kieda ${ }^{14}$, H. KrawCZynski ${ }^{3}$, F. Krennrich ${ }^{20}$, K. LeE ${ }^{3}$, A. S Madhavan ${ }^{20}$,

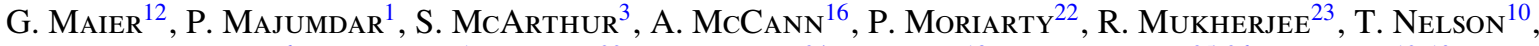

A. O'Faoláin de Bhróithe ${ }^{6}$, R. A. Ong ${ }^{1}$, M. OrR ${ }^{20}$, A. N. Otte ${ }^{24}$, N. PARK ${ }^{18}$, J. S. Perkins ${ }^{25,26}$, M. Pohl ${ }^{12,13}$, H. ProKoph $^{12}$, J. QuinN ${ }^{6}$, K. RagaN ${ }^{16}$, L. C. Reyes ${ }^{27}$, P. T. Reynolds ${ }^{28}$, E. RoaChe ${ }^{4}$, J. RuPPel ${ }^{12,13}$, D. B. SAXON ${ }^{17}$, M. Schroedter ${ }^{4}$, G. H. Sembroski ${ }^{9}$, C. Skole ${ }^{12}$, A. W. Smith ${ }^{14}$, I. Telezhinsky ${ }^{13,12}$, G. Tešić ${ }^{16}$, M. Theiling ${ }^{9}$, S. Thibadeaud $^{3}$, K. Tsurusaki $^{15}$, A. Varlotta ${ }^{9}$, M. Vivier ${ }^{17}$, S. P. Wakely ${ }^{18}$, J. E. WArd ${ }^{3}$, A. Weinstein ${ }^{20}$, R. Welsing ${ }^{12}$, D. A. Williams ${ }^{2}$, B. ZitZer ${ }^{5}$, C. PFrOMMER ${ }^{29}$, AND A. PINZKE ${ }^{30}$

${ }^{1}$ Department of Physics and Astronomy, University of California, Los Angeles, CA 90095, USA

${ }^{2}$ Santa Cruz Institute for Particle Physics and Department of Physics, University of California, Santa Cruz, CA 95064, USA ${ }^{3}$ Department of Physics, Washington University, St. Louis, MO 63130, USA

${ }^{4}$ Fred Lawrence Whipple Observatory, Harvard-Smithsonian Center for Astrophysics, Amado, AZ 85645, USA

${ }^{5}$ Argonne National Laboratory, 9700 S. Cass Avenue, Argonne, IL 60439, USA

${ }^{6}$ School of Physics, University College Dublin, Belfield, Dublin 4, Ireland

${ }^{7}$ School of Physics, National University of Ireland Galway, University Road, Galway, Ireland

${ }^{8}$ Astronomy Department, Adler Planetarium and Astronomy Museum, Chicago, IL 60605, USA

9 Department of Physics, Purdue University, West Lafayette, IN 47907, USA

${ }^{10}$ School of Physics and Astronomy, University of Minnesota, Minneapolis, MN 55455, USA

${ }^{11}$ Department of Astronomy and Astrophysics, 525 Davey Lab, Pennsylvania State University, University Park, PA 16802, USA

12 DESY, Platanenallee 6, D-15738 Zeuthen, Germany; pohlmadq@gmail.com

${ }^{13}$ Institute for Physics and Astronomy, University of Potsdam, D-14476 Potsdam, Germany

${ }^{14}$ Department of Physics and Astronomy, University of Utah, Salt Lake City, UT 84112, USA

${ }^{15}$ Department of Physics and Astronomy, University of Iowa, Van Allen Hall, Iowa City, IA 52242, USA

${ }^{16}$ Physics Department, McGill University, Montreal, QC H3A 2T8, Canada

${ }^{17}$ Department of Physics and Astronomy and The Bartol Research Institute, University of Delaware, Newark, DE 19716, USA

${ }^{18}$ Enrico Fermi Institute, University of Chicago, Chicago, IL 60637, USA

${ }^{19}$ Physics Department, Columbia University, New York, NY 10027, USA

${ }^{20}$ Department of Physics and Astronomy, Iowa State University, Ames, IA 50011, USA

${ }^{21}$ Department of Physics and Astronomy, DePauw University, Greencastle, IN 46135-0037, USA

${ }^{22}$ Department of Life and Physical Sciences, Galway-Mayo Institute of Technology, Dublin Road, Galway, Ireland

${ }^{23}$ Department of Physics and Astronomy, Barnard College, Columbia University, NY 10027, USA

${ }^{24}$ School of Physics \& Center for Relativistic Astrophysics, Georgia Institute of Technology, 837 State Street NW, Atlanta, GA 30332-0430, USA

${ }^{25}$ CRESST and Astroparticle Physics Laboratory NASA/GSFC, Greenbelt, MD 20771, USA

${ }^{26}$ Center for Space Science and Technology, University of Maryland, Baltimore County, 1000 Hilltop Circle, Baltimore, MD 21250, USA

${ }^{27}$ Physics Department, California Polytechnic State University, San Luis Obispo, CA 94307, USA

${ }^{28}$ Department of Applied Physics and Instrumentation, Cork Institute of Technology, Bishopstown, Cork, Ireland

${ }^{29}$ Heidelberg Institute for Theoretical Studies, Schloss-Wolfsbrunnenweg 35, D-69118 Heidelberg, Germany; christoph.pfrommer@h-its.org

${ }^{30}$ Department of Physics, University of California, Santa Barbara, CA 93106, USA

Received 2012 February 26; accepted 2012 July 12; published 2012 September 11

\section{ABSTRACT}

Observations of radio halos and relics in galaxy clusters indicate efficient electron acceleration. Protons should likewise be accelerated and, on account of weak energy losses, can accumulate, suggesting that clusters may also be sources of very high energy (VHE; $E>100 \mathrm{GeV}$ ) gamma-ray emission. We report here on VHE gamma-ray observations of the Coma galaxy cluster with the VERITAS array of imaging Cerenkov telescopes, with complementing Fermi Large Area Telescope observations at GeV energies. No significant gamma-ray emission from the Coma Cluster was detected. Integral flux upper limits at the $99 \%$ confidence level were measured to be on the order of $(2-5) \times 10^{-8}$ photons $\mathrm{m}^{-2} \mathrm{~s}^{-1}$ (VERITAS, $>220 \mathrm{GeV}$ ) and $\sim 2 \times 10^{-6}$ photons $\mathrm{m}^{-2} \mathrm{~s}^{-1}$ (Fermi, $1-3 \mathrm{GeV}$ ), respectively. We use the gamma-ray upper limits to constrain cosmic rays (CRs) and magnetic fields in Coma. Using an analytical approach, the CR-to-thermal pressure ratio is constrained to be $<16 \%$ from VERITAS data and $<1.7 \%$ from Fermi data (averaged within the virial radius). These upper limits are starting to constrain the CR physics in self-consistent cosmological cluster simulations and cap the maximum CR acceleration efficiency at structure formation shocks to be $<50 \%$. Alternatively, this may argue for non-negligible CR transport processes such as CR streaming and diffusion into the outer cluster regions. Assuming that the radio-emitting electrons of the Coma halo result from hadronic CR interactions, the observations imply a lower limit on the central magnetic field in Coma of $\sim(2-5.5) \mu \mathrm{G}$, depending on the radial magnetic field profile and on the gamma-ray spectral index. Since these values are below those inferred by Faraday rotation measurements in Coma (for most of the parameter space), this renders the hadronic model a very plausible explanation of the Coma radio halo. Finally, since galaxy clusters 
are dark matter (DM) dominated, the VERITAS upper limits have been used to place constraints on the thermally averaged product of the total self-annihilation cross section and the relative velocity of the DM particles, $\langle\sigma v\rangle$.

Key words: cosmic rays - dark matter - galaxies: clusters: general - galaxies: clusters: individual (Coma (ACO 1656)) - gamma rays: galaxies: clusters - magnetic fields

Online-only material: color figures

\section{INTRODUCTION}

Clusters of galaxies are the largest virialized objects in the universe, with typical sizes of a few Mpc and masses on the order of $10^{14}-10^{15} M_{\odot}$. According to the currently favored hierarchical model of cosmic structure formation, larger objects formed through successive mergers of smaller objects with galaxy clusters sitting on top of this mass hierarchy (see Voit 2005 for a review). Most of the mass $(\sim 80 \%)$ in a cluster is dark matter (DM), as indicated by galaxy dynamics and gravitational lensing (Diaferio et al. 2008). Baryonic gas making up the intracluster medium (ICM) contributes about $15 \%$ of the total cluster mass, and individual galaxies account for the remainder (about $5 \%$ ). The ICM gas mass also composes a significant fraction of the observable (baryonic) matter in the universe.

The ICM is a hot $\left(T \sim 10^{8} \mathrm{~K}\right)$ plasma-emitting thermal bremsstrahlung in the soft X-ray regime (see, e.g., Petrosian 2001). This plasma has been heated primarily through collisionless structure formation shocks that form as a result of the hierarchical merging and accretion processes. Such shocks and turbulence in the ICM gas in combination with intra-cluster magnetic fields also provide a means to accelerate particles efficiently (see, e.g., Colafrancesco \& Blasi 1998; Ryu et al. 2003). Many clusters feature megaparsec-scale halos of nonthermal radio emission, indicative of a population of relativistic electrons and magnetic fields permeating the ICM (Cassano et al. 2010). There are two competing theories to explain radio halos. In the "hadronic model," the radio-emitting electrons and positrons are produced in inelastic collisions of cosmic-ray (CR) ions with the thermal gas of the ICM (Dennison 1980; Enßlin et al. 2011). In the "re-acceleration model," a long-lived pool of $100 \mathrm{MeV}$ electrons-previously accelerated by formation shocks, galactic winds, or jets of active galactic nuclei (AGNs) - interacts with plasma waves that are excited during states of strong ICM turbulence, e.g., after a cluster merger. This may result in second-order Fermi acceleration and may produce energetic electrons $(\sim 10 \mathrm{GeV})$ sufficient to explain the observable radio emission (Schlickeiser et al. 1987; Brunetti \& Lazarian 2011). Observations of possibly nonthermal emission from clusters in the extreme-ultraviolet (EUV; Sarazin \& Lieu 1983) and hard X-rays (Rephaeli \& Gruber 2002; Fusco-Femiano et al. 2004; Eckert et al. 2007) may provide further indication of relativistic particle populations in clusters, although the interpretation of these observations as nonthermal diffuse emission has been disputed on the basis of more sensitive observations (see, e.g., Ajello et al. 2009, 2010; Wik et al. 2009).

Galaxy clusters have, for many years, been proposed as sources of gamma rays. If shock acceleration in the ICM is an efficient process, a population of highly relativistic CR protons and heavy ions is to be expected in the ICM. The main energy-loss mechanism for $\mathrm{CR}$ hadrons at high energies is pion production through the interaction of CRs with nuclei in the ICM. Pions are short-lived and decay. The decay of neutral pions produces gamma rays, and the decay of charged pions produces muons, which then decay to electrons and positrons. Due to the low density of the ICM $\left(n_{\mathrm{ICM}} \sim 10^{-3} \mathrm{~cm}^{-3}\right)$, the large size and the volume-filling magnetic fields in the ICM, CR hadrons will be confined in the cluster on timescales comparable to, or longer than, the Hubble time (Völk et al. 1996; Berezinsky et al. 1997), and they can therefore accumulate. For a given CR distribution function, the hadronically induced gamma-ray flux is directly proportional to the CR-to-thermal pressure fraction, $X_{\mathrm{CR}}=\left\langle P_{\mathrm{CR}}\right\rangle /\left\langle P_{\mathrm{th}}\right\rangle$ (see, e.g., Enßlin et al. 2007), where the brackets indicate volume averages. A very modest $X_{\mathrm{CR}}$ of a few percent implies an observable flux of gamma rays (e.g., Pfrommer \& Enßlin 2004a).

Hydrostatic estimates of cluster masses, which are determined by balancing the thermal pressure force and the gravitational force, are biased low by the presence of any substantial nonthermal pressure component, including a CR pressure contribution. Similarly, a substantial CR pressure can bias the temperature decrement of the cosmic microwave background (CMB) due to the Sunyaev-Zel'dovich effect in the direction of a galaxy cluster. This could then severely jeopardize the use of clusters to determine cosmological parameters. Comparing X-ray and optical potential profiles in the centers of galaxy clusters yields an upper limit of $20 \%-30 \%$ of nonthermal pressure (that can be composed of CRs, magnetic fields, or turbulence) relative to the thermal gas pressure (Churazov et al. 2008, 2010). An analysis that compares spatially resolved weak gravitational lensing and hydrostatic X-ray masses for a sample of 18 galaxy clusters detects a deficit of the hydrostatic mass estimate compared to the lensing mass of $20 \%$ at $R_{500}$ - the radius within which the mean density is 500 times the critical density of the universesuggesting again a substantial nonthermal pressure contribution on large scales (Mahdavi et al. 2008). Observing gamma-ray emission is a complementary method of constraining the pressure contribution of $\mathrm{CRs}$ that is most sensitive to the cluster core region. However, it assumes that the $\mathrm{CR}$ component is fully mixed with the ICM and may not allow for a detection of a two-phase structure of CRs and the thermal ICM. An $X_{\mathrm{CR}}$ of only a few percent is required in order to produce a gammaray flux observable with the current generation of gamma-ray telescopes, rendering this technique at least as sensitive as the dynamical and hydrostatic methods (which are more general in that they are sensitive to any nonthermal pressure component).

Gamma-ray emission can also be produced by Compton upscattering of ambient photons, for example, CMB photons, on ultrarelativistic electrons. Those electrons can be either secondaries from the $\mathrm{CR}$ interactions mentioned above or injected into the ICM by powerful cluster members and further accelerated by diffusive shock acceleration or turbulent reacceleration processes (Schlickeiser et al. 1987, and references therein).

A third mechanism for gamma-ray production in a galaxy cluster could be self-annihilation of a DM particle, e.g., a weakly interacting massive particle (WIMP). As already mentioned, about $80 \%$ of the cluster mass is in the form of DM, which makes 
galaxy clusters interesting targets for DM searches (Evans et al. 2004; Bergström \& Hooper 2006; Pinzke et al. 2009; Cuesta et al. 2011) despite their large distances compared to other common targets for DM searches, such as dwarf spheroidal galaxies (Strigari et al. 2007; Acciari et al. 2010; Aliu et al. 2009) or the Galactic center (Kosack et al. 2004; Aharonian et al. 2006, 2009a; Abramowski et al. 2011).

While several observations of clusters of galaxies have been made with satellite-borne and ground-based gamma-ray telescopes, a detection of gamma-ray emission from a cluster has yet to be made. Observations with EGRET (Sreekumar et al. 1996; Reimer et al. 2003) and the Large Area Telescope (LAT) on board the Fermi Gamma-ray Space Telescope (Ackermann et al. 2010) have provided upper limits on the gamma-ray fluxes (typically $\sim 10^{-9}$ photons $\mathrm{cm}^{-2} \mathrm{~s}^{-1}$ for Fermi-LAT observations) for several galaxy clusters in the $\mathrm{MeV}$ to $\mathrm{GeV}$ band. Upper limits on the very high energy (VHE) gamma-ray flux from a small sample of clusters, including the Coma Cluster, have been provided by observations with ground-based imaging atmospheric Cerenkov telescopes (IACTs; Perkins et al. 2006; Perkins 2008; A haronian et al. 2009b; Aleksić et al. 2010, 2012).

The Coma Cluster of galaxies (ACO 1656) is one of the most thoroughly studied clusters across all wavelengths (Voges et al. 1999). Located at a distance of about $100 \mathrm{Mpc}(z=0.023$; Struble \& Rood 2003), it is one of the closest massive clusters ( $M \sim 10^{15} M_{\odot}$; Smith 1998; Kubo et al. 2008). It hosts both a giant radio halo (Giovannini et al. 1993; Thierbach et al. 2003) and peripheral radio relic, which appears connected to the radio halo with a "diffuse" bridge (see discussion in Brown \& Rudnick 2011). It has been suggested (Enßlin et al. 1998) and successively demonstrated by cosmological simulations, which model the nonthermal emission processes (Pfrommer et al. 2008; Pfrommer 2008; Battaglia et al. 2009; Skillman et al. 2011), that the relic could well be an infall shock. Extended soft thermal X-ray emission is evident from the ROSAT all-sky survey in the $0.1-2.4 \mathrm{keV}$ band (Briel et al. 1992). Observations with XMM-Newton (Briel et al. 2001) revealed substructure in the X-ray halo supported by substantial turbulent pressure of at least $\sim 10 \%$ of the total pressure (Schuecker et al. 2004). The Coma Cluster is a natural candidate for gamma-ray observations.

In this article, results from the VERITAS observations of the Coma Cluster of galaxies are reported, with complementing analysis of available data from the LAT on board the Fermi Gamma-ray Space Telescope. The VERITAS and Fermi-LAT data have been used to place constraints on CR particle populations, magnetic fields, and DM in the cluster. Throughout the analyses, a present-day Hubble constant of $H_{0}=100 \mathrm{~h} \mathrm{~km} \mathrm{~s}-1$ $\mathrm{Mpc}^{-1}$ with $h=0.7$ has been used.

\section{VERITAS OBSERVATIONS, ANALYSIS, AND RESULTS}

The VERITAS gamma-ray detector (Weekes et al. 2002) is an array of four $12 \mathrm{~m}$ diameter IACTs (Holder et al. 2006) located at an altitude of $\sim 1250 \mathrm{~m}$ a.s.l. at the Fred Lawrence Whipple Observatory in southern Arizona $\left(31^{\circ} 40^{\prime} 30^{\prime \prime} \mathrm{N}, 110^{\circ} 57^{\prime} 07^{\prime \prime} \mathrm{W}\right)$. Each of the telescopes is equipped with a 499 pixel camera covering a 3.5 field of view. The array, completed in the fall of 2007, is designed to detect gamma-ray emission from astrophysical objects in the energy range from $100 \mathrm{GeV}$ to more than $30 \mathrm{TeV}$. Depending on the zenith angle and quality selection criteria imposed during the data analysis, the effective energy range may be narrower than that. The energy resolution is $\sim 15 \%$, and the angular resolution ( $68 \%$ containment) is $\sim 0.1$ per event at $1 \mathrm{TeV}$ and slightly larger at low energy. At the time of the Coma Cluster observations, the sensitivity of the array allowed for detection of a point source with a flux of $1 \%$ of the steady Crab Nebula flux above $300 \mathrm{GeV}$ at the confidence level of five standard deviations $(5 \sigma)$ in under $45 \mathrm{hr}^{31}$

The Coma Cluster was observed with VERITAS between March and May in 2008 with all four telescopes fully operational. The total exposure amounts to $18.6 \mathrm{hr}$ of quality-selected live time, i.e., time periods of astronomical darkness with clear sky conditions and no technical problems with the array. The center of the cluster was tracked in wobble mode, where the expected source location is offset from the center of the field of view by $0.5 \mathrm{deg}$, to allow for simultaneous background estimation (Fomin et al. 1994). All of the observations were made in a small range with average zenith angle $\sim 21^{\circ}$.

The data analysis was performed following the standard VERITAS procedures described in Cogan et al. (2008) and Daniel et al. (2008). Prior to event reconstruction and selection, all shower images are calibrated and cleaned. Showers are then reconstructed for events with at least two telescopes contributing images that pass the following quality selection criteria: more than four participating pixels in the camera, number of photoelectrons in the image larger than 75, and the distance from the image centroid to the center of the camera less than 1.43 . These quality selection criteria impose an energy threshold $^{32}$ of about $220 \mathrm{GeV}$. In addition, events for which only images from the two closest-spaced telescopes ${ }^{33}$ survive quality selection are rejected, as they introduce an irreducible high background rate due to local muons, degrading the instrument sensitivity (Maier \& Knapp 2007).

Gamma-ray-like events are separated from the CR background by imposing selection criteria (cuts) on the meanscaled length and width parameters (Aharonian et al. 1997; Krawczynski et al. 2006) calculated from a parameterized moment analysis of the shower images (Hillas 1985). These parameters are averages over the four telescopes weighted with the total amplitude of the images, which measure the image moment width and length scaled with values expected for gamma rays. In this analysis, events with a mean-scaled length in the range 0.05-1.19 and a mean-scaled width in the range 0.05-1.08 are selected as gamma-ray-like events. These ranges for the gammahadron separation cuts were optimized a priori for a weak point source (3\% Crab Nebula flux level) and a differential spectral index of 2.4, using data taken on the Crab Nebula during the same epoch. Because the VHE gamma-ray spectrum for the Coma Cluster is expected to be a power-law function with an index of about 2.3 (Pinzke \& Pfrommer 2010), these cuts are suitable for the analysis of the Coma Cluster data set. It is noted that slightly varying the spectral index $( \pm 0.2)$ does not significantly impact the cuts used for quality selection and gamma-hadron separation in this work.

The Coma Cluster is a very rich cluster of galaxies with many plausible sites for gamma-ray emission: the core region, the peripheral radio relic, and individual powerful cluster member galaxies. VERITAS has a large enough field of view to allow investigation of several of these scenarios. In this work, the focus has been on the core region and three cluster members.

\footnotetext{
31 The integral flux sensitivity above $300 \mathrm{GeV}$ was improved by about $30 \%$ with the relocation of one telescope in the summer of 2009.

32 The energy threshold is defined as the energy corresponding to the maximum of the product function of the observed spectrum and the collection area. It does not vary significantly for the different source scenarios and assumed spectral indices reported in this work.

33 In the array configuration prior to summer 2009, two telescopes had a separation of only $35 \mathrm{~m}$.
} 

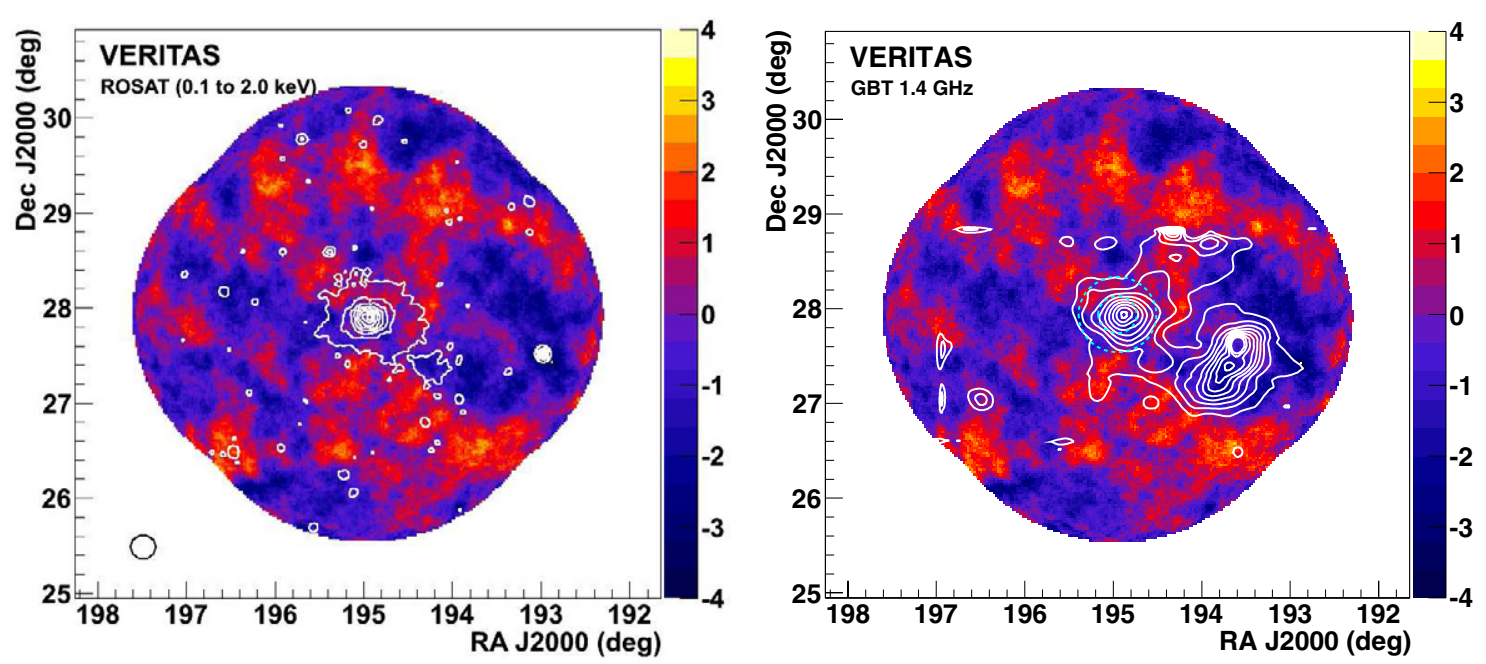

Figure 1. Left: smoothed significance map of the Coma Cluster calculated from the excess VHE gamma-ray events observed with VERITAS over a $4.5 \times 4.5$ field of view. The color scale indicates significance in units of standard deviations. The excess counts were derived using a ring-background model (Aharonian et al. 2001). White contours show the X-ray counts per second in the $0.1-2 \mathrm{keV}$ energy band (eight levels from 1 to 16 counts s $\mathrm{s}^{-1}$ after 3 pixel Gaussian smoothing) from the ROSAT all-sky survey (Briel et al. 1992). Right: same as the left panel but with overlaid contours (20-180 mJ in $20 \mathrm{~mJ}$ steps) from GBT radio observations at $1.4 \mathrm{GHz}$ (Brown \& Rudnick 2011), where strong point sources have been subtracted. Also shown are the 0.2 and 0.4 radii (dashed cyan) considered for the extended-source analyses presented here.

(A color version of this figure is available in the online journal.)

Table 1

Regions of Interest in the Coma Cluster Field of View

\begin{tabular}{lcr}
\hline \hline Source & $\begin{array}{c}\text { R.A. } \\
(\mathrm{J} 2000)\end{array}$ & \multicolumn{1}{c}{$\begin{array}{c}\text { Decl. } \\
(\mathrm{J} 2000)\end{array}$} \\
\hline Core & $12^{\mathrm{h}} 59^{\mathrm{m}} 48.7$ & $+27^{\circ} 58^{\prime} 50^{\prime \prime} .0$ \\
NGC 4889 & $13^{\mathrm{h}} 00^{\mathrm{m}} 08^{\mathrm{s}} .13$ & $+27^{\circ} 58^{\prime} 37^{\prime \prime} .03$ \\
NGC 4874 & $12^{\mathrm{h}} 59^{\mathrm{m}} 35^{\mathrm{s}} .71$ & $+27^{\circ} 57^{\prime} 33^{\prime \prime} .37$ \\
NGC 4921 & $13^{\mathrm{h}} 01^{\mathrm{m}} 26^{\mathrm{s}} .12$ & $+27^{\circ} 53^{\prime} 09^{\prime \prime} .59$ \\
\hline
\end{tabular}

Notes. The cluster core is considered as both a point source and a modestly extended source. Three central galaxies are also considered in point-source searches. The choice is based on evidence for an excess of nonthermal X-ray emission (Neumann et al. 2003) at the location of these galaxies.

The core region is treated as either a point source or a mildly extended source, a uniform disk with intrinsic radius 0.2 or 0.4 , similar to the extension of the thermal soft X-ray emission from the core. There is evidence of a recent merger event between the two central galaxies NGC 4889 and NGC 4874 (Tribble 1993). There is also evidence for an excess of nonthermal X-ray emission from these galaxies, as well as from the galaxy NGC 4921 (Neumann et al. 2003). Therefore, searches for point-like VHE gamma-ray emission have been conducted at the locations of these galaxies. The regions of interest considered in this work are summarized in Table 1.

The ring-background model (Aharonian et al. 2001) is used to estimate the background due to CRs misinterpreted as gamma rays (the cuts described above reject more than $99 \%$ of all CRs). The total number of events in a given region of interest (ROI) is then compared to the estimated background from the OFFsource region scaled by the ratio of the solid angles to produce a final excess or deficit. The VHE gamma-ray significance is then calculated according to Formula (17) in $\mathrm{Li} \& \mathrm{Ma}$ (1983). Significance skymaps over the VERITAS field of view produced with a 0.2 integration radius are shown in Figure 1 with overlaid X-ray and radio contours from the ROSAT all-sky survey (Briel et al. 1992) and Green Bank Telescope (GBT) 1.4 GHz observations (Brown \& Rudnick 2011), respectively.
Depending on the assumed extent of the source and the pointspread function (PSF), we can define an ON region, into which a defined fraction of the source photons should fall. No significant excess of VHE gamma rays from the Coma Cluster was detected with VERITAS, as illustrated by the $\theta^{2}$ distribution shown in Figure 2, in which source events would pile up at small values of $\theta^{2}$ for a point source and fall into a somewhat wider range of $\theta^{2}$ values for an extended source. The $\theta^{2}$ distribution is a plot of event density versus the square of the angular separation from a given location. It permits a comparison of the ON-source event distribution with that of other locations, in this case a ringshaped region, into which only background events should fall, the so-called OFF-source region. The $\theta^{2}$ distribution extends out to $0.42 \mathrm{deg}^{2}$ to cover both the case of point-like and extended emission from the core of the Coma Cluster. The $\theta^{2}$ distributions for the member galaxies also considered in this work are very similar to that in Figure 2 and show no excess of gamma rays. A 99\% confidence level upper limit is calculated for each $\mathrm{ROI}$ using events from the ON-source and OFF-source regions and the method described by Rolke et al. (2005) assuming a Gaussian-distributed background. A lower bound of zero is imposed on the gamma-ray flux from the Coma Cluster, which prevents artificially low flux upper limits in the case that the best-fit source flux is formally negative. Figure 3 shows the distribution of significances over the VERITAS skymap, which is well fit by a Gaussian with a mean close to zero and a standard deviation within a few percent of unity.

Table 2 lists the upper limits for the selected regions of interest shown in Table 1. These upper limit calculations depend on the gamma-ray spectrum, which in this work is assumed to be a power law in energy, $d N / d E \propto E^{-\alpha}$, where the spectral index $\alpha$ was allowed to have a value of $2.1,2.3$, or 2.5 .

\section{FERMI-LAT ANALYSIS AND RESULTS}

LAT on board Fermi has observed the Coma Cluster in allsky survey mode since its launch in 2008 June. Fermi-LAT is sensitive to gamma rays in the $20 \mathrm{MeV}$ to $\sim 300 \mathrm{GeV}$ energy range and is complementary to the VERITAS observations. 


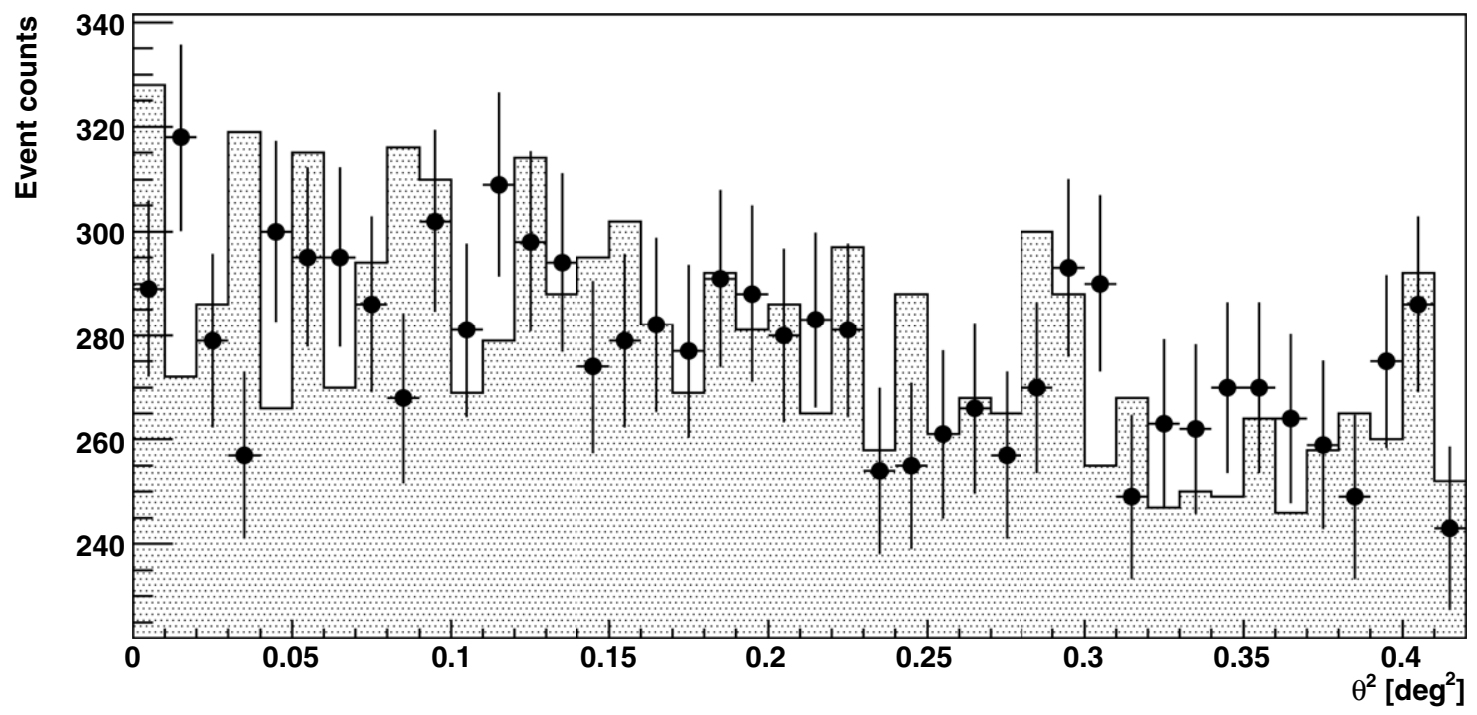

Figure 2. $\theta^{2}$ distribution from VERITAS observations of the Coma Cluster of galaxies. The points with error bars represent the ON-source data sample, and the filled area is the background estimation based on the OFF-source regions. Each bin represents an annulus around the Coma Cluster core position, and the annuli are all of equal area. The data were derived from the ring-background model using a 0.2 integration radius.

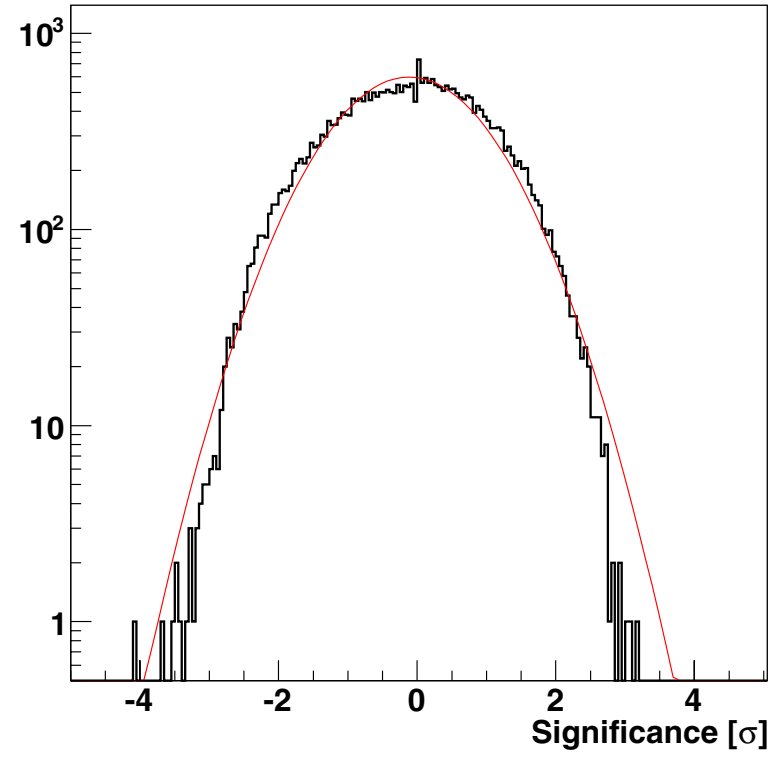

Figure 3. Distribution of significances for Figure 1 and an integration radius of 0.2 . The curve is a Gaussian fit to the data, with mean $\mu=-0.11 \pm 0.0059$ and standard deviation $\sigma=1.01 \pm 0.003$, which is consistent with the absence of gamma-ray sources in the field of view.

(A color version of this figure is available in the online journal.)

Ackermann et al. (2010) reported on the search for gammaray emission from 33 galaxy clusters in the data from the first 18 months, including the Coma Cluster, for which an upper limit of $4.58 \times 10^{-9}$ photons $\mathrm{cm}^{-2} \mathrm{~s}^{-1}$ in the $0.2-100 \mathrm{GeV}$ energy band was reported. This limit is expected to improve as the exposure is increased. In this work, an updated analysis is presented as a complement to the VERITAS results, which includes data taken between 2008 August 5 and 2012 April 17.

The LAT-data analysis of this work follows the same procedure as described in detail in Nolan et al. (2012) and was performed with the Fermi Science Tools version 9.23.1. To only include events with high probability of being photons, the P7SOURCE class and the corresponding P7SOURCE_V6 instrument-response functions were used throughout this work.
A zenith-angle cut of $100^{\circ}$ was applied to eliminate albedo gamma rays from Earth's limb, excluding time intervals during which any part of the ROI was outside the field of view. In addition, time intervals were removed during which the observatory was transiting the Southern Atlantic Anomaly or the rocking angle exceeded $52^{\circ}$.

The ROI is defined to be a square region of the sky measuring $14^{\circ}$ on a side and centered on $\alpha_{J 2000}=194.953$ and $\delta_{J 2000}=$ 27.9806, the nominal position of the Coma Cluster.

Only photons with reconstructed energy greater than $1 \mathrm{GeV}$ are considered, for which the $68 \%$ containment radius of the PSF is narrower than $\sim 0.8$. The Fermi-LAT Collaboration estimates the systematic uncertainties on the effective area at $10 \mathrm{GeV}$ to be around $10 \% .{ }^{34}$

The background emission in the ROI was modeled using 14 point sources listed in the second LAT source catalog (Nolan et al. 2012), the LAT standard Galactic diffuse emission component (gal_2yearp7v6_v0.fit), and the corresponding isotropic template (iso_p7v6source.txt) that accounts for extragalactic emission and residual CR contamination. Due to the large tails of the PSF at low energy, a further 14 point sources, lying $\sim 4^{\circ}$ outside the ROI, were included in the source model.

The energy spectra of 24 sources are described by a power law. The remaining four sources, ${ }^{35}$ being bright sources, are modeled with additional degrees of freedom using the lognormal representation, which is typically used for modeling blazar spectra.

The analysis is performed in three energy bins: $1-3 \mathrm{GeV}$, 3-10 GeV, and 10-30 GeV. To find the best-fit spectral parameters, a binned maximum-likelihood analysis (Mattox et al. 1996) is performed for each energy bin on a map with 0.1 pixel size in gnomonic (onto a tangent plane) projection, covering the entire ROI. To determine the significance of the sources, and in particular that of the Coma Cluster, the analysis tool uses the likelihood-ratio test statistic (TS; Mattox et al. 1996) defined as

$$
\mathrm{TS}=-2\left(\ln L_{0}-\ln L\right),
$$

\footnotetext{
34 http://fermi.gsfc.nasa.gov/ssc/data/analysis/LAT_caveats.html 35 2FGLJ1303.1+2435, 2FGLJ1310.6+3222, 2FGLJ1226.0+2953, and 2FGLJ1224.9+2122.
} 
Table 2

VERITAS VHE Gamma-ray Flux Upper Limits for Different Regions of Interest in the Coma Cluster of Galaxies and Surroundings

\begin{tabular}{|c|c|c|c|c|c|c|c|c|c|}
\hline \multirow[t]{2}{*}{ Source } & \multirow[t]{2}{*}{$R^{\mathrm{a}}(\mathrm{deg})$} & \multirow[t]{2}{*}{$N_{S}^{\mathrm{b}}$} & \multirow[t]{2}{*}{$S^{\mathrm{c}}(\sigma)$} & \multicolumn{6}{|c|}{ Flux UL ${ }^{d}$} \\
\hline & & & & \multicolumn{2}{|c|}{$\alpha=2.1$} & \multicolumn{2}{|c|}{$\alpha=2.3$} & \multicolumn{2}{|c|}{$\alpha=2.5$} \\
\hline \multirow[t]{3}{*}{ Core } & 0 & 17 & 0.84 & 2.59 & $(0.78 \%)$ & 2.78 & $(0.83 \%)$ & 2.97 & $(0.89 \%)$ \\
\hline & 0.2 & -41 & -1.0 & 1.96 & $(0.59 \%)$ & 2.09 & $(0.63 \%)$ & 2.21 & $(0.66 \%)$ \\
\hline & 0.4 & -26 & -0.30 & 4.44 & $(1.3 \%)$ & 4.74 & $(1.4 \%)$ & 5.02 & $(1.5 \%)$ \\
\hline NGC 4889 & 0 & 3 & 0.14 & $\ldots$ & $\ldots$ & 1.85 & $(0.55 \%)$ & $\ldots$ & $\ldots$ \\
\hline NGC 4874 & 0 & -14 & -0.71 & $\ldots$ & $\ldots$ & 1.51 & $(0.45 \%)$ & $\ldots$ & $\ldots$ \\
\hline NGC 4921 & 0 & -4 & -0.23 & $\ldots$ & $\ldots$ & 2.41 & $(0.72 \%)$ & $\ldots$ & $\ldots$ \\
\hline
\end{tabular}

Notes.

${ }^{a}$ Intrinsic source radius (zero means point source), which is convolved with the gamma-ray point-spread function.

b Net event counts in the source region.

${ }^{\mathrm{c}}$ Statistical significance calculated according to Li \& Ma (1983).

d $99 \%$ confidence level upper limit in units of $10^{-8}$ photons $\mathrm{m}^{-2} \mathrm{~s}^{-1}$ calculated according to Rolke et al. (2005) above an energy threshold of $220 \mathrm{GeV}$, with corresponding fluxes in percent of the steady Crab Nebula flux in parentheses, for different values of the spectral index, $\alpha$.

Table 3

Fermi-LAT HE Gamma-ray Flux Upper Limits for the Coma Cluster Core

\begin{tabular}{|c|c|c|c|}
\hline Spatial Model & $\begin{array}{c}1-3 \mathrm{GeV} \\
\text { Flux } \mathrm{UL}^{\mathrm{a}} \text { (Significance) }\end{array}$ & $\begin{array}{c}3-10 \mathrm{GeV} \\
\text { Flux } \mathrm{UL}^{\mathrm{a}} \text { (Significance) }\end{array}$ & $\begin{array}{c}10-30 \mathrm{GeV} \\
\text { Flux } \mathrm{UL}^{\mathrm{a}} \text { (Significance) }\end{array}$ \\
\hline \multicolumn{4}{|c|}{ Spectral index $\alpha=2.1$} \\
\hline Point source & $1.882(0.000)$ & $0.759(0.000)$ & $0.671(0.830)$ \\
\hline Disk: $r=0.2$ & $2.109(0.152)$ & $0.899(0.000)$ & $0.719(0.740)$ \\
\hline Disk: $r=0.4$ & $2.438(0.201)$ & $1.232(0.619)$ & $0.875(1.387)$ \\
\hline \multicolumn{4}{|c|}{ Spectral index $\alpha=2.3$} \\
\hline Point source & $1.946(0.000)$ & $0.788(0.000)$ & $0.667(0.874)$ \\
\hline Disk: $r=0.2$ & $2.180(0.169)$ & $0.941(0.000)$ & $0.725(0.828)$ \\
\hline Disk: $r=0.4$ & $2.524(0.246)$ & $1.275(0.742)$ & $0.869(1.390)$ \\
\hline \multicolumn{4}{|c|}{ Spectral index $\alpha=2.5$} \\
\hline Point source & $2.008(0.000)$ & $0.816(0.000)$ & $0.663(0.915)$ \\
\hline Disk: $r=0.2$ & $2.246(0.189)$ & $0.979(0.020)$ & $0.720(0.864)$ \\
\hline Disk: $r=0.4$ & $2.606(0.291)$ & $1.313(0.856)$ & $0.861(1.387)$ \\
\hline
\end{tabular}

Note. ${ }^{a} 99 \%$ confidence level flux upper limit in units of $10^{-6}$ photons $\mathrm{m}^{-2} \mathrm{~s}^{-1}$.

where $L_{0}$ is the maximum-likelihood value for the null hypothesis and $L$ is the maximum likelihood with the additional source at a given position on the sky.

In the likelihood analysis, the spatial parameters of the sources were kept fixed at the values given in the catalog, whereas the spectral parameters of the point sources in the ROI, along with the normalization of the diffuse components, were allowed to freely vary. We analyzed three cases in which the gamma-ray emission from the Coma Cluster was assumed to follow a powerlaw spectrum with a photon index $\alpha=2.1,2.3$, and 2.5. The spectral indices of all point sources were permitted to freely vary between $\alpha=0$ and $\alpha=5$. We considered the emission as being caused by both a point-like and a spatially extended source (a uniform disk) with radius $r=0.2$ or $r=0.4$, as in the VERITAS analysis.

No significant gamma-ray signal was detected. For one free parameter, the flux from the Coma Cluster, the detection significance is computed as the square root of the test statistic (TS follows a $\chi_{1}^{2}$ distribution). The highest TS was obtained for the high-energy (HE) band, where TS $\sim 0.8$ for the point source model, TS $\sim 0.7$ for the disk model with $r=0.2$, and TS $\sim 2$ for the disk model with $r=0.4$.

We therefore used the profile likelihood method (Rolke et al. 2005 ) to derive flux upper limits at the $99 \%$ confidence level in the energy range 1-30 GeV, assuming both an unresolved, point-like and spatially extended emission, as shown in Table 3.

\section{GAMMA-RAY EMISSION FROM COSMIC RAYS}

We decided to adopt a multifaceted approach to constrain the CR-to-thermal pressure distribution in the Coma Cluster using the upper limits derived from the VERITAS and FermiLAT data in this work. This approach includes (1) a simplified multi-frequency analytical model that assumes a constant CR-to-thermal energy density and a power-law spectrum in momentum, (2) an analytic model derived from cosmological hydrodynamical simulations of the formation of galaxy clusters, and (3) a model that uses the observed intensity profile of the giant radio halo in Coma to place a lower limit on the expected gamma-ray flux in the hadronic model-where the radio-emitting electrons are secondaries from $\mathrm{CR}$ interactions and which is independent of the magnetic field distribution. This last approach translates into a minimum CR pressure, which, if challenged by tight gamma-ray limits/detections, permits scrutiny of the hadronic interaction model of the formation of giant radio halos. Alternatively, realizing a spatial CR distribution that is consistent with the flux upper limits, and requiring the model to match the observed radio data, enables us to 
derive a lower limit on the magnetic field distribution. We stress again that this approach assumes the validity of the hadronic interaction model. Modeling the CR distribution through different techniques enables us to bracket our lack of understanding about the underlying plasma physics that shapes the CR distribution and hence to reflect on the Bayesian priors that are imposed on the modeling (see Pinzke et al. 2011 for a discussion).

\subsection{Simplified Analytical Model}

We start by adopting a simplified analytical model that assumes a power-law CR spectrum and a constant CR-tothermal pressure ratio, i.e., we adopt the isobaric model of CRs following the approach of Pfrommer \& Enßlin (2004a). To be independent of additional assumptions and in line with earlier work in the literature, we do not impose a low-momentum cutoff, $q$, on the CR distribution function, i.e., we adopt $q=0$. Since, a priori, the CR spectral index is unconstrained, ${ }^{36}$ we vary it in the range $2.1<\alpha<2.5$, which is compatible with the radio spectral index of the giant radio halo of the Coma Cluster after accounting for the spectral steepening at frequencies $v \sim 5 \mathrm{GHz}$ due to the Sunyaev-Zel'dovich effect (Enßlin 2002; Pfrommer \& Enßlin 2004a). ${ }^{37}$ To model the thermal pressure, we adopt the electron density profile for the Coma Cluster that has been inferred from ROSAT X-ray observations (Briel et al. 1992) and use a constant temperature of $k T=8.25 \mathrm{keV}$ throughout the virial region.

Table 4 shows the resulting constraints on the CR-to-thermal pressure ratio, $X_{\mathrm{CR}}=\left\langle P_{\mathrm{CR}}\right\rangle /\left\langle P_{\mathrm{th}}\right\rangle$, averaged within the virial radius, $R_{\text {vir }}=2.2 \mathrm{Mpc}$, which we define as the radius of a sphere enclosing a mean density that is 200 times the critical density of the universe. Constraints on $X_{\mathrm{CR}}$ with VERITAS flux upper limits (99\% CL) strongly depend on $\alpha$. This is due to the comparably large energy range from $\mathrm{GeV}$ energies (which dominate the CR pressure, provided that $\alpha>2$ and the $\mathrm{CR}$ population has a nonrelativistic low-momentum cutoff, i.e., $q<m_{p} c$, where $m_{p}$ is the proton mass) to energies at $220 \mathrm{GeV}$, where our quality selection criteria imposed the energy threshold. These gamma-ray energies correspond to $1.6 \mathrm{TeV}$ $\mathrm{CRs}$ - an energy ratio of more than three orders of magnitude, which explains the sensitivity to small changes in $\alpha$. The flux measurements within 0.2 are the most constraining due to a competition between the integrated signal and the background as the integration radius increases. This yields limits on $X_{\mathrm{CR}}$ between 0.048 and 0.43 (for $\alpha$ varying between 2.1 and 2.5), with a constraint of $X_{\mathrm{CR}}<0.1$ for $\alpha=2.3$ (close to the spectral index predicted by the simulations of Pinzke \& Pfrommer (2010) around $220 \mathrm{GeV}$ ). Constraints on $X_{\mathrm{CR}}$ with Fermi-LAT limits (99\% CL) depend only weakly on $\alpha$ because GeV-band gamma rays are produced by $\mathrm{CRs}$ with energies near the relativistic transition, which dominantly contribute to the CR pressure. $X_{\mathrm{CR}}$ constraints with Fermi-LAT limits are most constraining for an aperture of 0.4 ; despite the slightly weaker flux upper limits in comparison to the smaller radii of integration, we expect a considerably larger gamma-ray luminosity due to the increasing volume in this model. The best limit of $X_{\mathrm{CR}}<0.012$ is achieved for $\alpha=2.3$, while the limit for $\alpha=2.1$ is only slightly worse $\left(X_{\mathrm{CR}}<0.017\right)$.

\footnotetext{
36 The hadronic interaction physics guarantees that the CR spectral index coincides with that of the resulting pion-decay gamma-ray emission at energies $E \gg 1 \mathrm{GeV}$ that are well above the pion bump (see discussion in Pfrommer \& Enßlin 2004a).

37 Assuming a magnetic field of $1 \mu \mathrm{G}$, the CR protons responsible for the $\mathrm{GHz}$ radio-emitting electrons have an energy of $\sim 100 \mathrm{GeV}$ and are $\sim 20$ times less energetic than those $\mathrm{CR}$ protons responsible for $200 \mathrm{GeV}$ gamma-ray emission.
}

Table 4

Constraints on the CR-to-thermal Pressure Ratio in the Coma Cluster Core (Simplified, Isobaric Analytic Model) for Different Spatial Extensions and Predicted Fluxes for the Energy Bands $1-3 \mathrm{GeV}$ and $>220 \mathrm{GeV}$ (Simulation-based Model)

\begin{tabular}{lllllc}
\hline \hline$R^{\mathrm{a}}(\mathrm{deg})$ & \multicolumn{3}{c}{ Analytic Model: $X_{\mathrm{CR}}{ }^{\mathrm{b}}$} & $F_{\gamma, \operatorname{sim}}(E)^{\mathrm{c}}$ & $F_{\mathrm{UL}} / F_{\gamma, \operatorname{sim}}(>E)^{\mathrm{d}}$ \\
\cline { 2 - 4 } & $\alpha=2.1$ & $\alpha=2.3$ & $\alpha=2.5$ \\
\hline \multicolumn{5}{c}{ VERITAS constraints } \\
\hline 0 & 0.1 & 0.23 & 0.97 & 1.9 & 14.8 \\
0.2 & 0.048 & 0.10 & 0.43 & 2.9 & 7.2 \\
0.4 & 0.067 & 0.15 & 0.62 & 4.4 & 10.8 \\
\hline \multicolumn{7}{c}{ Fermi constraints } \\
\hline 0 & 0.035 & 0.024 & 0.033 & 1.4 & 1.34 \\
0.2 & 0.024 & 0.017 & 0.022 & 2.1 & 1.00 \\
0.4 & 0.017 & 0.012 & 0.016 & 3.2 & 0.76 \\
\hline
\end{tabular}

Notes.

a Intrinsic source radius (zero means point source), which is convolved with the gamma-ray point-spread function.

${ }^{\mathrm{b}}$ Constraint on the CR-to-thermal pressure ratio, $X_{\mathrm{CR}}=\left\langle P_{\mathrm{CR}}\right\rangle /\left\langle P_{\mathrm{th}}\right\rangle$, which was assumed to be constant throughout the cluster and calculated according to Pfrommer \& Enßlin (2004a).

${ }^{c}$ Integrated gamma-ray flux from the simulation-based analytic model by Pinzke \& Pfrommer (2010): above $E=220 \mathrm{GeV}$ in units of $10^{-9}$ photons $\mathrm{m}^{-2} \mathrm{~s}^{-1}$ for VERITAS and for $E=1-3 \mathrm{GeV}$ in units of $10^{-6}$ photons $\mathrm{m}^{-2} \mathrm{~s}^{-1}$ for Fermi.

d Ratio of flux upper limit $\left(F_{\mathrm{UL}}\right)$ to integrated gamma-ray flux from the simulation-based analytic model, with the UL based on spectral index $\alpha=2.3$ in the VERITAS band $(E>220 \mathrm{GeV})$ and $\alpha=2.1$ in the Fermi band $(E=1-3 \mathrm{GeV})$.

\subsection{Simulation-based Approach}

We complement the simplified analytical analysis with a more realistic and predictive approach derived from cosmological hydrodynamical simulations. We adopt the universal spectral and spatial gamma-ray model developed by Pinzke \& Pfrommer (2010) to estimate the emission from decaying neutral pions, which in clusters dominates over the inverse Compton (IC) emission above $100 \mathrm{MeV}$. Given a density profile as, e.g., inferred by cosmological simulations or X-ray observations, the analytic approach models the $\mathrm{CR}$ distribution and the associated radiative emission processes from radio to the gamma-ray band. This formalism was derived from high-resolution simulations of clusters of galaxies that included radiative hydrodynamics, star formation, and supernova feedback, and it followed the CR physics by tracing the most important injection and loss processes self-consistently while accounting for the CR pressure in the equation of motion (Pfrommer et al. 2006; Enßlin et al. 2007; Jubelgas et al. 2008). The results are in line with earlier numerical results on some of the overall characteristics of the CR distribution and the associated radiative emission processes (Dolag \& Enßlin 2000; Miniati et al. 2001; Miniati 2003; Pfrommer et al. 2007, 2008; Pfrommer 2008).

The overall normalization of the CR and gamma-ray distribution scales nonlinearly with the acceleration efficiency at structure formation shocks. Following recent observations of supernova remnants (Helder et al. 2009), as well as theoretical studies (Kang \& Jones 2005), we adopt an optimistic but nevertheless realistic value of this parameter and assume that $50 \%$ of the dissipated energy at strong shocks is injected into CRs, with this efficiency decreasing rapidly for weaker shocks. Since the vast majority of internal formation shocks (merger and flow shocks) are weak shocks with Mach numbers $M \lesssim 3$ (e.g., 


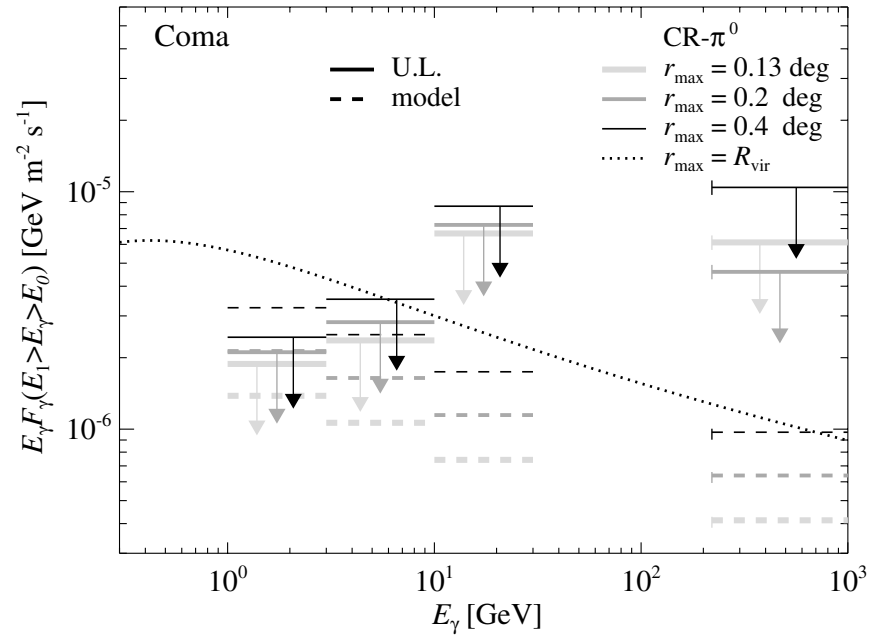

Figure 4. Integral gamma-ray flux upper limits in narrow energy bands $\left[E_{0} ; E_{1}\right]$ for Fermi and VERITAS observations of the Coma Cluster (given in Tables 2 and 3 ) for different integration radii (arrows with different gray intensities), assuming that $\alpha=2.3$ except in the $1-3 \mathrm{GeV}$ energy interval, where $\alpha=2.1$ is adopted. $R_{\text {vir }}$ is the virial radius of the Coma Cluster, corresponding to 1 . 25 . These are compared to integrated spectra of the same energy interval and aperture, assuming the universal gamma-ray spectrum of clusters (lines with different gray intensities; Pinzke \& Pfrommer 2010). To guide the eye, we show the underlying universal integral energy distribution of pion-decay gamma rays, $E_{\gamma} F_{\gamma}\left(>E_{\gamma}\right)$, resulting from hadronic interactions of CRs and ICM protons $\left(\mathrm{CR}-\pi^{0}\right.$, dotted). For visualization purposes, all photon fluxes are weighted with the smallest energy in each interval. Note that the Fermi limit for the energy interval of $1-3 \mathrm{GeV}$ within the aperture of 0.4 is the most constraining.

Ryu et al. 2003), they do not contribute significantly to the CR population in clusters. Instead, strong shocks during the formation epoch of clusters and strong accretion shocks at the present time (at the boundary of voids and filaments/supercluster regions) dominate the acceleration of CRs that are adiabatically transported through the cluster. Hence, the model provides a plausible upper limit for the CR contribution from structure formation shocks in galaxy clusters, which can be scaled with the effective acceleration efficiency. Other possible CR sources, such as AGNs and starburst-driven galactic winds, have been neglected for simplicity but could in principle increase the expected gamma-ray yield.

These cosmological simulations only consider advective transport of CRs by bulk gas flows that inject a turbulent cascade, leading to centrally enhanced density profiles. However, other means of CR transport such as diffusion and streaming may flatten the CR radial profiles. The CRs stream along magnetic field lines in the opposite direction of the CR number density gradient (at any energy). In the stratified cluster atmosphere, this implies a net flux of CRs toward larger radii, equalizing the CR number density with time if not counteracted by advective transport. It has been suggested that advection velocities only dominate over the CR streaming velocities for periods with trans- and supersonic cluster turbulence during a cluster merger and drop below the CR streaming velocities for relaxing clusters. As a consequence, a bimodality of the CR spatial distribution is expected to result, with merging (relaxed) clusters showing a centrally concentrated (flat) CR energy density profile (Enßlin et al. 2011). This translates into a bimodality of the expected diffuse radio and gamma-ray emission of clusters, since more centrally concentrated CRs will find higher target densities for hadronic $\mathrm{CR}$ proton interactions. As a result of this, relaxed clusters could have a reduced gamma-ray luminosity by up to a factor of five (Enßlin et al. 2011). Hence, tight upper limits

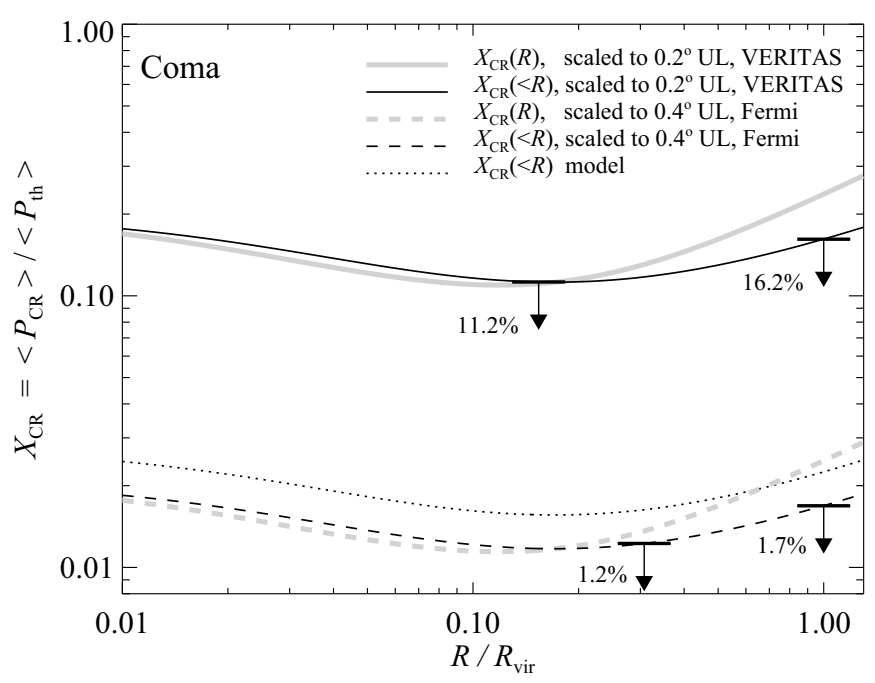

Figure 5. CR-to-thermal pressure ratio, $X_{\mathrm{CR}}=\left\langle P_{\mathrm{CR}}\right\rangle /\left\langle P_{\mathrm{th}}\right\rangle$, as a function of radial distance from the center of the Coma Cluster. The model predictions, shown by dashed curves (Pinzke \& Pfrommer 2010), have been scaled to match the most constraining VERITAS upper limits within 0.2 (solid) and Fermi upper limits within 0.4 (dashed). We compare differential $X_{\mathrm{CR}}$ profiles (gray) to integrated profiles $X_{\mathrm{CR}}\left(<R / R_{\mathrm{vir}}\right)=\int_{0}^{R} P_{\mathrm{CR}} d V / \int_{0}^{R} P_{\mathrm{th}} d V$ (black), which we use to compare to the upper limits.

on the gamma-ray emission can constrain a combination of acceleration physics and transport properties of CRs.

We adopt the density profile of thermal electrons as discussed in Section 4.1 and model the temperature profile of the Coma Cluster with a constant central temperature of $k T=8.25 \mathrm{keV}$ and a characteristic decline toward the cluster periphery in accordance with a fit to the universal profile derived from cosmological cluster simulations (Pinzke \& Pfrommer 2010; Pfrommer et al. 2007) and the behavior of a nearby sample of deep Chandra cluster data (Vikhlinin et al. 2005). This enables us to adopt the spatial and spectral distribution of CRs according to the model by Pinzke \& Pfrommer (2010) that neglects the contribution of supernova remnants, AGNs, and cluster galaxies.

Figure 4 shows the expected integral spectral energy distribution of Coma within the virial radius (dotted line). This suggests a spectral index of $\alpha=2.1$ in the energy interval $1-3 \mathrm{GeV}$ and $\alpha=2.3$ for energies probed by VERITAS (>220 GeV). Also shown are integrals of the differential spectrum for finite energy intervals across the angular apertures tested in this study (dashed lines). These model fluxes (summarized in Table 4) are compared to Fermi and VERITAS flux upper limits for the same energy intervals. Constraints on $X_{\mathrm{CR}}$ with the gamma-ray flux limit of Fermi in the energy interval $1-3 \mathrm{GeV}(<0.4)$ are most constraining, since that combination of a specific energy interval and aperture minimizes the ratio of the upper limit to the expected model flux. In particular, this upper limit is $24 \%$ below the model predictions that assume an optimistically large shock acceleration efficiency and CR transport parameters as laid out above. Hence, this enables us to constrain a combination of maximum shock acceleration efficiency and CR transport parameters. In our further analysis, we use the most constraining Fermi-LAT flux limits in the energy interval $1-3 \mathrm{GeV}$, as well as the gamma-ray flux limits of VERITAS in the energy range above $220 \mathrm{GeV}$.

Figure 5 shows the CR-to-thermal pressure ratio, $X_{\mathrm{CR}}=$ $\left\langle P_{\mathrm{CR}}\right\rangle /\left\langle P_{\mathrm{th}}\right\rangle$, as a function of radial distance, $R$, from the Coma Cluster center and contained within $R$. All radii are shown in units of the virial radius, $R_{\mathrm{vir}}=2.2 \mathrm{Mpc}$. To compute the 
CR pressure, we assume a low-momentum cutoff of the CR distribution at $q=0.8 m_{p} c$, where $m_{p}$ is the proton mass. This is suggested by cosmological cluster simulations and reflects the high Coulomb cooling rates at low CR energies. The CR-tothermal pressure ratio rises toward the outer regions on account of the higher efficiency of CR acceleration at the peripheral accretion shocks compared to the weak central flow shocks. Adiabatic compression of a mixture of CRs and thermal gas disfavors the $\mathrm{CR}$ pressure relative to the thermal pressure on account of the softer equation of state of CRs. The weak increase of $X_{\mathrm{CR}}$ toward the core is due to the comparably fast thermal cooling of gas.

In the case of VERITAS, for the most constraining regions tested (within an aperture of radius 0.2 ), the predicted CR pressure is a factor of 7.2 below the inferred upper limits of VERITAS (see Table 2 and assuming a spectral index of $\alpha=2.3$, which matches the simulated one at energies $E_{\gamma}=200 \mathrm{GeV}$ ). To first order, we can scale the averaged CR-to-thermal pressure ratio of our model by that factor, keep the spatial behavior, and obtain an integrated limit of the CR-tothermal pressure ratio of $X_{\mathrm{CR}}<0.112$ within 0.2 that translates to a limit within the cluster virial radius of $X_{\mathrm{CR}}<0.162$ (solid lines of Figure 5). This limit is less constraining by $50 \%$ in comparison to the simplified analytical model, which gives $X_{\mathrm{CR}}<0.1$. This difference is explained by the concavity of the simulated spectrum, which therefore carries more pressure at $\mathrm{GeV}$ energies than a pure power-law spectrum with $\alpha=2.3$.

As already alluded to, the most constraining Fermi-LAT upper limit in the energy interval $1-3 \mathrm{GeV}(<0.4)$ is a factor of 0.76 smaller than our model predictions (assuming $\alpha=2.1$, which is very close to the simulated spectral index for the energy range 1-3 GeV). Scaling our integrated CR-to-thermal pressure profile yields a constraint of $X_{\mathrm{CR}}<0.012$ within 0.4 that translates to a limit within the cluster virial radius of $X_{\mathrm{CR}}<0.017$ (dashed lines of Figure 5). The $X_{\mathrm{CR}}$ constraint evaluated within the cluster virial radius is comparable to the constraint of $X_{\mathrm{CR}}<0.017$ in our simplified model. Naturally, with the Fermi-LAT limits we probe the region around $\mathrm{GeV}$ energies that dominate the CR pressure, and we do not expect any differences to the simplified power-law model in comparison to the universal $\mathrm{CR}$ spectrum with its concave $\mathrm{CR}$ spectrum found in the simulations.

\subsection{Minimum Gamma-Ray Flux}

For clusters that host radio halos, we can derive a minimum gamma-ray flux in the hadronic model of radio halos-where the radio-emitting electrons are secondaries from CR interactions. Hadronic interactions channel about the same power into secondary electrons and $\pi^{0}$-decay gamma rays. A stationary distribution of CR electrons loses all its energy to synchrotron radiation for strong magnetic fields $\left(B \gg B_{\mathrm{CMB}} \simeq(1+z)^{2} 3.2 \mu \mathrm{G}\right.$, where $B_{\mathrm{CMB}}$ is the equivalent magnetic field strength of the $\mathrm{CMB}$, so that $B_{\mathrm{CMB}}^{2} / 8 \pi$ equals the CMB energy density). Thus, the ratio of gamma ray to synchrotron flux becomes independent of the spatial distribution of CRs and thermal gas (Völk 1989; Pohl 1994; Pfrommer 2008), in particular with $\alpha_{v} \simeq 1$ as the observed synchrotron spectral index. Hence, we can derive a minimum gamma-ray flux in the hadronic model

$$
F_{\gamma, \text { min }}=\frac{A_{\gamma}}{A_{v}} \frac{L_{v}}{4 \pi D_{\text {lum }}^{2}},
$$

where $L_{v}$ is the observed luminosity of the radio mini-halo, $D_{\text {lum }}$ denotes the luminosity distance to the respective cluster,
Table 5

Minimum Gamma-ray Fluxes in the Hadronic Model of Radio Halos, Where the Radio-emitting Electrons Are Secondaries from CR Interactions, and Corresponding Minimum CR-to-thermal Pressure Ratios for Coma

\begin{tabular}{|c|c|c|c|c|c|c|}
\hline \multirow[t]{2}{*}{$R^{\mathrm{a}}(\mathrm{deg})$} & \multicolumn{3}{|c|}{$F_{\gamma, \min }(>E)^{\mathrm{b}}$} & \multicolumn{3}{|c|}{$10^{4} \times X_{\mathrm{CR}, \min ^{\mathrm{c}}}$} \\
\hline & $\alpha=2.1$ & $\alpha=2.3$ & $\alpha=2.5$ & $\alpha=2.1$ & $\alpha=2.3$ & $\alpha=2.5$ \\
\hline \multicolumn{7}{|c|}{ VERITAS energy range, $E>220 \mathrm{GeV}$} \\
\hline 0 & 1.6 & 0.7 & 0.3 & 6.7 & 6.1 & 11 \\
\hline 0.2 & 3.1 & 1.4 & 0.6 & 7.8 & 7.2 & 13 \\
\hline 0.4 & 6.3 & 2.8 & 1.3 & 9.8 & 9.0 & 16 \\
\hline \multicolumn{7}{|c|}{ Fermi energy range, $1-3 \mathrm{GeV}$} \\
\hline 0 & 3.5 & 4.8 & 6.4 & 6.7 & 6.1 & 11 \\
\hline 0.2 & 6.8 & 9.3 & 12.5 & 7.8 & 7.2 & 13 \\
\hline 0.4 & 13.5 & 18.6 & 25.0 & 9.8 & 9.0 & 16 \\
\hline
\end{tabular}

Notes.

${ }^{a}$ Intrinsic source radius (zero means point source), which is convolved with the gamma-ray point-spread function.

b Minimum gamma-ray flux derived from the hadronic model described in Section 4.3. Values are in units of $10^{-10}$ photons $\mathrm{m}^{-2} \mathrm{~s}^{-1}$ for the VERITAS energy range and $10^{-8}$ photons $\mathrm{m}^{-2} \mathrm{~s}^{-1}$ for the Fermi energy range.

c Minimum CR-to-thermal pressure ratio, $X_{\mathrm{CR}, \min }$, in the hadronic model described in Section 4.3. For simplicity, we duplicate $X_{\mathrm{CR} \text {, min }}$ for the VERITAS and Fermi constraints: for a given realization of the CR pressure (and a magnetic field model that is trivial here as we assume $B \gg B_{\mathrm{CMB}}$ ), we can derive the radio flux and gamma-ray fluxes in various bands ( $1-3 \mathrm{GeV},>220 \mathrm{GeV})$.

and $A_{\gamma}$ and $A_{v}$ are dimensional constants that depend on the hadronic physics of the interaction (Pfrommer 2008; Pfrommer et al. 2008). Lowering the magnetic field would require an increase in the energy density of CR electrons to reproduce the observed synchrotron luminosity and thus increase the associated gamma-ray flux.

To derive a minimum gamma-ray flux that can be compared to the upper limits, we need to determine the radio flux within the corresponding angular regions. To this end, we fit the pointsource-subtracted, azimuthally averaged radio-halo profile at $1.38 \mathrm{GHz}$ (Deiss et al. 1997) with a $\beta$-model

$$
S_{v}\left(r_{\perp}\right)=S_{0}\left[1+\left(\frac{r_{\perp}}{r_{\mathrm{c}}}\right)^{2}\right]^{-3 \beta+1 / 2},
$$

where $S_{0}=1.1 \times 10^{-3} \mathrm{Jy} \mathrm{arcmin}^{-2}, r_{\mathrm{c}}=450 \mathrm{kpc}$, and $\beta=0.78$. Within the error bars, this profile is consistent with $326 \mathrm{MHz}$ data taken by Govoni et al. (2001) when scaled with a radio spectral index of 1.15 .

The results for the minimum gamma-ray flux $F_{\gamma, \min }$ $(>220 \mathrm{GeV})$ and the minimum CR-to-thermal pressure ratio $X_{\mathrm{CR} \text {, min }}=X_{\mathrm{CR}} F_{\gamma, \min } / F_{\gamma \text {,iso }}$ are shown in Table 5, where $F_{\gamma \text {,iso }}$ is the gamma-ray flux in the simplified model introduced in Section 4.1. Even in the most constraining cases, and assuming $\alpha \leqslant 2.3$, these are a factor of $\sim 60$ below the VERITAS upper limits (for $\alpha=2.1,<0.2$ ) and a factor of $\sim 20$ below the Fermi-LAT upper limits (for $\alpha=2.3,<0.4$ ). Note that these minimum gamma-ray fluxes are sensitive to the variation of the CR proton spectral index with energy as a result of, for example, momentum-dependent diffusion. Assuming a plausible value for the central magnetic field of Coma of $5 \mu \mathrm{G}$ (Bonafede et al. 2010), the radio-halo emission at $\mathrm{GHz}$ frequencies is dominated by electrons with energy $E_{\mathrm{e}} \sim 2.5 \mathrm{GeV}$ (which corresponds to proton energies $E_{\mathrm{p}} \sim 40 \mathrm{GeV}$ ). Gamma rays with an energy of $200 \mathrm{GeV}$ are produced by $\mathrm{CR}$ protons with an energy of $E_{\mathrm{p}} \sim 1.6 \mathrm{TeV}-$ a factor of 50 higher than 
those probed by radio-halo observations. A steepening of the CR proton spectral index of 0.2 between $40 \mathrm{GeV}$ and $1.6 \mathrm{TeV}$ would imply a decrease in the minimum gamma-ray flux by a factor of two.

\subsection{Constraining the Magnetic Field}

In the previous section, we have obtained an absolute lower limit on the gamma-ray emission in the hadronic model by assuming high magnetic fields, $B \gg B_{\mathrm{CMB}}$. We can turn the argument around and use our upper limit on the gamma-ray emission (and by extension on the CR pressure) to infer a lower limit on the magnetic field needed to explain the observed radio emission. This, again, assumes the validity of the hadronic model of radio halos, in which the radio-emitting electrons are secondaries from $\mathrm{CR}$ interactions. A stronger gamma-ray constraint will tighten the magnetic field limit. In case of a conflict with magnetic field measurements by other methods, e.g., Faraday rotation measure (RM) ${ }^{38}$ the hadronic model of radio halos would be challenged. The method we use to constrain the magnetic field inherits a dependence on the assumed radial scaling, which we parameterize as

$$
B(r)=B_{0}\left(\frac{n_{\mathrm{e}}(r)}{n_{\mathrm{e}}(0)}\right)^{\alpha_{B}},
$$

as suggested by Faraday RM studies and numerical magnetohydrodynamical (MHD) simulations (Bonafede et al. 2010; Bonafede et al. 2011, and references therein). Here $n_{\mathrm{e}}$ denotes the Coma electron density profile (Briel et al. 1992). In fact, the magnetic field in the Coma Cluster is among the best constrained, because its proximity permits RM observations of seven radio sources located at projected distances of $50-1500 \mathrm{kpc}$ from the cluster center. The best-fit model yields $B_{0}=4.7_{-0.8}^{+0.7} \mu \mathrm{G}$ and $\alpha_{B}=0.5_{-0.1}^{+0.2}$ (Bonafede et al. 2010). We aim to constrain the central field strength, $B_{0}$, and we permit the magnetic decline, $\alpha_{B}$, to vary within a reasonable range of $\Delta \alpha_{B}=0.2$ as suggested by those Faraday RM studies. We proceed as follows:

1. Given a model for the magnetic field with $\alpha_{B}$ and an initial guess for $B_{0}$, we determine the profile of the CR-to-thermal pressure ratio, $X_{\mathrm{CR}}(r)$, by matching the hadronically produced synchrotron emission to the observed radio-halo emission over the entire extent. To this end, we deproject the fit to the surface brightness profile of Equation (3) (using an Abel integral equation; see the Appendix of Pfrommer $\&$ Enßlin 2004a), yielding the radio emissivity

$$
\begin{aligned}
j_{v}(r) & =\frac{S_{0}}{2 \pi r_{\mathrm{c}}} \frac{6 \beta-1}{\left(1+r^{2} / r_{\mathrm{c}}^{2}\right)^{3 \beta}} \mathcal{B}\left(\frac{1}{2}, 3 \beta\right) \\
& =j_{v, 0}\left(1+r^{2} / r_{\mathrm{c}}^{2}\right)^{-3 \beta},
\end{aligned}
$$

where $\mathcal{B}$ denotes the beta function. It is generically true for weak magnetic fields $\left(B<B_{\mathrm{CMB}}\right)$ in the outer parts of the Coma halo that the product $X_{\mathrm{CR}}(r) X_{B}(r)$ (where $X_{B}$ denotes the magnetic-to-thermal energy density ratio) has to increase by a factor of about 100 toward the radio-halo periphery to account for the observed extent. If we were to adopt a steeper magnetic decline such as $\alpha_{B}=0.5$, which produces a flat $X_{B}(r)$, the CR-to-thermal pressure ratio would have to rise accordingly by a factor of 100 .

\footnotetext{
38 Generally, Faraday RM analyses of the magnetic field strength by, e.g., background sources observed through clusters are degenerate with the magnetic coherence scale and may be biased by the unknown correlation between magnetic and density fluctuations.
}

Table 6

Constraints on Magnetic Fields in the Hadronic Model of the Coma Radio Halo and the Corresponding CR-to-thermal Pressure Ratio (at the Largest

\begin{tabular}{|c|c|c|c|c|c|c|}
\hline \multirow[b]{3}{*}{$\alpha_{B}$} & \multicolumn{6}{|c|}{ Minimum Magnetic Field, $B_{0, \min }(\mu \mathrm{G})^{\mathrm{a}}$} \\
\hline & \multicolumn{3}{|c|}{ VERITAS Constraints } & \multicolumn{3}{|c|}{ Fermi Constraints } \\
\hline & $\alpha=2.1$ & $\alpha=2.3$ & $\alpha=2.5$ & $\alpha=2.1$ & $\alpha=2.3$ & $\alpha=2.5$ \\
\hline 0.3 & 0.69 & 0.57 & 0.48 & 1.38 & 1.95 & 2.68 \\
\hline 0.5 & 0.97 & 0.80 & 0.68 & 1.94 & 2.74 & 3.78 \\
\hline 0.7 & 1.40 & 1.17 & 0.99 & 2.80 & 3.97 & 5.50 \\
\hline \multicolumn{7}{|c|}{ Corresponding $X_{\mathrm{CR}}(1 \mathrm{Mpc})$} \\
\hline 0.3 & 0.46 & 1.05 & 4.55 & 0.11 & 0.08 & 0.11 \\
\hline 0.5 & 0.74 & 1.70 & 7.47 & 0.18 & 0.13 & 0.17 \\
\hline 0.7 & 1.09 & 2.59 & 11.55 & 0.27 & 0.19 & 0.26 \\
\hline
\end{tabular}
Emission Radius of $1 \mathrm{Mpc}$ ) Such That the Model Reproduces the Observed Radio Surface-brightness Profile

Notes. ${ }^{\text {a }}$ The parameters of the magnetic field are the magnetic decline, $\alpha_{B}$, and the central field strength, $B_{0}$, which are defined by $B(r)=B_{0}\left[n_{\mathrm{e}}(r) / n_{\mathrm{e}}(0)\right]^{\alpha_{B}}$. In all cases, we used the most constraining $R=0.4$; see Section 4.4 for details.

2. Given this realization for $X_{\mathrm{CR}}$, we compute the pion-decay gamma-ray surface brightness profile, integrate the flux within a radius of $(0.13,0.2,0.4) \mathrm{deg}$, and scale the CR profile in order to match the corresponding VERITAS/ Fermi flux upper limits. This scaling factor, $X_{\mathrm{CR}, 0}$, depends on the CR spectral index, $\alpha$ (assuming a power-law $\mathrm{CR}$ population for simplicity), the radial decline of the magnetic field, $\alpha_{B}$, and our initial guess for $B_{0}$.

3. We then solve for $B_{0}$ while matching the observed synchrotron profile and fixing the profile of $X_{\mathrm{CR}}(r)$ as determined through the previous two steps. Note that for $B_{0} \gg B_{\mathrm{CMB}}$ and a radio spectral index of $\alpha_{v}=1$, the solution would be degenerated since the luminosity of the radio halo scales as

$$
L_{v} \propto \int d V Q(E) \frac{B^{1+\alpha_{v}}}{B^{2}+B_{\mathrm{CMB}}^{2}} \rightarrow \int d V Q(E),
$$

where $Q(E)$ denotes the electron source function.

4. IC cooling of $\mathrm{CR}$ electrons on $\mathrm{CMB}$ photons introduces a characteristic scale of $B_{\mathrm{CMB}} \simeq 3.2 \mu \mathrm{G}$, which imprints as a nonlinearity on the synchrotron emissivity as a function of magnetic field strength (see Equation (6)). Hence, we have to iterate through the previous steps until our solution for the minimum magnetic field $B_{0}$ converges.

Table 6 shows the resulting lower limit of the central magnetic field ranging from $B_{0}=0.5$ to $1.4 \mu \mathrm{G}$ in the case of VERITAS and from $B_{0}=1.4$ to $5.5 \mu \mathrm{G}$ in the case of Fermi-LAT. ${ }^{39}$ Since these lower limits on $B_{0}$ are below the values favored by Faraday RM for most of the parameter space spanned by $\alpha_{B}$ and $\alpha$ (and never exceed the values for the phenomenological Faraday RM-inferred $B$-model), the hadronic model is a viable explanation of the Coma radio halo. In fact, the Fermi-LAT upper limits start to rule out the parameter combination of $\alpha_{B} \gtrsim 0.7$ and $\alpha \gtrsim 2.5$ for the hadronic model of the Coma radio halo. Future gamma-ray observations of the Coma Cluster may put more stringent constraints on the parameters of the hadronic model.

A few remarks are in order. (1) For the VERITAS limits, the hardest CR spectral indices correspond to the tightest limits

\footnotetext{
39 Note that a central magnetic field of $3 \mu \mathrm{G}$ corresponds in the Coma Cluster to a magnetic-to-thermal energy density ratio of $X_{B}=0.005$.
} 
on $B_{0}$, because the CR flux is constrained around $1 \mathrm{TeV}$ and a comparably small fraction of CRs at $100 \mathrm{GeV}$ would be available to produce radio-emitting electrons. A high magnetic field would be required to match the observed synchrotron emission. The opposite is true for the Fermi upper limits at $1 \mathrm{GeV}$, which probe CRs around a pivot point of $8 \mathrm{GeV}$ : a soft CR spectral index implies a comparably small fraction of CRs at $100 \mathrm{GeV}$, and hence a strong magnetic field is needed to match the observed synchrotron flux. (2) For a steeper magnetic decline (larger $\alpha_{B}$ ), the CR number density needs to be larger to match the observed radio-emission profiles, which would yield a higher gamma-ray flux so that the upper limits are more constraining. This implies tighter lower limits for $B_{0}$. (3) Interestingly, in all cases, the 0.4 aperture limits are the most constraining. For a given magnetic realization, a substantially increasing CR-tothermal pressure profile is needed to match the observed radio profiles, and therefore that $\mathrm{CR}$ realization produces a larger flux within 0.4 in comparison with the simplified CR model ( $X_{\mathrm{CR}}=$ const.), for which the 0.2 aperture limits are more constraining in the case of VERITAS. Physically, the large CR pressure in the cluster periphery may arise from CR streaming into the large available phase space in the outer regions.

As a final word, in Table 6 we show the corresponding values for the CR-to-thermal pressure ratio (at the largest emission radius at $1 \mathrm{Mpc}$ ) such that the model reproduces the observed radio surface brightness profile. ${ }^{40}$ They should be interpreted as upper limits since they are derived from flux upper limits. For the Fermi-LAT upper limits, they range from 0.08 to 0.27 ; hence, the $X_{\mathrm{CR}}$ profiles always obey the energy condition, i.e., $P_{\mathrm{CR}}<P_{\mathrm{th}}$, over the entire range of the radio-halo emission $(<1 \mathrm{Mpc}){ }^{41}$ The corresponding values for $X_{\mathrm{CR}}$ in the cluster center are smaller than 0.01 for the entire parameter space probed in this study. We conclude that the hadronic model is not challenged by current Faraday RM data and is a perfectly viable possibility in explaining the Coma radio-halo emission.

\section{EMISSION FROM DARK-MATTER ANNIHILATIONS}

As already mentioned in the introduction, most of the mass in a galaxy cluster is in the form of DM. While the nature of DM remains unknown, a compelling theoretical candidate is a WIMP. The self-annihilation of WIMPs can produce either monoenergetic gamma-ray lines or a continuum of secondary gamma rays that deviates significantly from the power-law spectra observed from most conventional astrophysical sources, with a sharp cutoff at the WIMP mass. These spectral features, together with the expected difference in the intensity distribution compared to conventional astrophysical sources, allow a clear, indirect detection of DM.

The expected gamma-ray flux due to self-annihilation of WIMPs in a DM halo is given by

$$
\frac{d \Phi_{\gamma}(\Delta \Omega, E)}{d E}=\frac{\langle\sigma v\rangle}{8 \pi m_{\chi}^{2}} \frac{d N_{\gamma}}{d E} J(\Delta \Omega),
$$

\footnotetext{
40 Note that in this section, we determine the radial behavior of $X_{\mathrm{CR}}$ by adopting a specific model for the magnetic field and requiring the modeled synchrotron surface brightness profile to match the observed data of the Coma radio halo. This is in contrast to the simplified analytical CR model, where $X_{\mathrm{CR}}$ is constant (Section 4.1), and to the simulation-based model, where $X_{\mathrm{CR}}(r)$ is derived from cosmological cluster simulations (Section 4.2).

41 See Figure 3 in Pfrommer \& Enßlin (2004b) for the entire parameter range assuming minimum-energy conditions, and Pfrommer \& Enßlin (2004a), Figure 7 for a parameterization as adopted in this study. We caution, however, that the minimum-energy condition is violated at the outer radio-halo boundary for the range of minimum magnetic field values inferred by this study.
}

where $\langle\sigma v\rangle$ is the thermally averaged product of the total selfannihilation cross section and the relative WIMP velocity, $m_{\chi}$ is the WIMP mass, $d N_{\gamma} / d E$ is the differential gamma-ray yield per annihilation, ${ }^{42} \Delta \Omega$ is the observed solid angle, and $J$ is the so-called astrophysical factor-a factor that determines the DM annihilation rate and depends on the DM distribution.

Given the upper limit on the observed gamma-ray rate, defined as the ratio of the event number detected within the observing time $T_{\text {obs }}, R_{\gamma}(99 \% \mathrm{CL})=N_{\gamma}(99 \% \mathrm{CL}) / T_{\text {obs }}$, we can place constraints on the WIMP parameter space $\left(m_{\chi},\langle\sigma v\rangle\right)$. Integrating Equation (7) over energy, we find

$$
\begin{aligned}
\langle\sigma v\rangle(99 \% \mathrm{CL})< & R_{\gamma}(99 \% \mathrm{CL}) \frac{8 \pi m_{\chi}^{2}}{J(\Delta \Omega)} \\
\times & {\left[\int_{0}^{m_{\chi}} d E A_{\mathrm{eff}} \frac{d N_{\gamma}(E)}{d E}\right]^{-1}, }
\end{aligned}
$$

where $A_{\text {eff }}$ is the effective area of the gamma-ray detector. Because the self-annihilation of a WIMP is a two-body process, the astrophysical factor $J(\Delta \Omega)$ is the line-of-sight integral of the DM density squared

$$
J(\Delta \Omega)=\int_{\Delta \Omega} d \Omega \int d \lambda \rho_{\chi}^{2}(\lambda, \Omega),
$$

where $\lambda$ represents the line of sight. In this work, we have modeled the Coma DM distribution with a Navarro, Frenk, and White (NFW) profile (Navarro et al. 1997),

$$
\rho_{\chi}(r)=\rho_{s}\left(\frac{r}{r_{s}}\right)^{-1}\left(1+\frac{r}{r_{s}}\right)^{-2},
$$

where $r_{s}$ is the scale radius and $\rho_{s}$ is the scale density. Using weak-lensing measurements of the virial mass in the Coma Cluster and the DM halo mass-concentration relation derived from $\mathrm{N}$-body simulation of structure formation (Bullock et al. 2001), Gavazzi et al. (2009) find, and list in their Table 1 (note that they define $\left.R_{\text {vir }}=R_{100}\right), M_{\text {vir }}=M_{200}=9.7(+6.1 /-3.5)$. $10^{14} h^{-1} M_{\odot}$ and $C_{\text {vir }}=C_{200}=3.5(+1.1 /-0.9)$, which we translate into the density-profile parameters $r_{s}=0.654 \mathrm{Mpc}$ and $\rho_{s}=4.4 \times 10^{14} M_{\odot} \mathrm{Mpc}^{-3}$. Note that the uncertainties are not necessarily distributed as a Gaussian and also arise from the choice of DM profile. According to the latest highresolution DM-only simulations of nine rich galaxy clusters, the inner regions of the smooth density profiles are quite well approximated by the NFW formula (Gao et al. 2012). However, gravitational interactions of DM with baryons may modify these predictions. This could give rise to either an increasing inner density slope due to adiabatic contraction of the DM component in response to cooling baryons in the central regions or a decreasing density slope due to violent baryonic feedback processes pushing gas out of the center by, e.g., energy injection through AGNs. However, on scales $r \gtrsim 0.45 \mathrm{Mpc}$ or more than $20 \%$ of $R_{\text {vir }}$ (which are of relevance for the present work), different assumptions about the inner slope of the smooth DM density profile translate to uncertainties in the resulting astrophysical factor. Table 7 lists the astrophysical factors calculated for the different VERITAS apertures considered in this work. Table 7 also lists the astrophysical factor calculated for the background region, which is used to estimate the gamma-ray

\footnotetext{
42 In this work, we have calculated the differential gamma-ray yield per annihilation using the Pythia Monte Carlo simulator.
} 


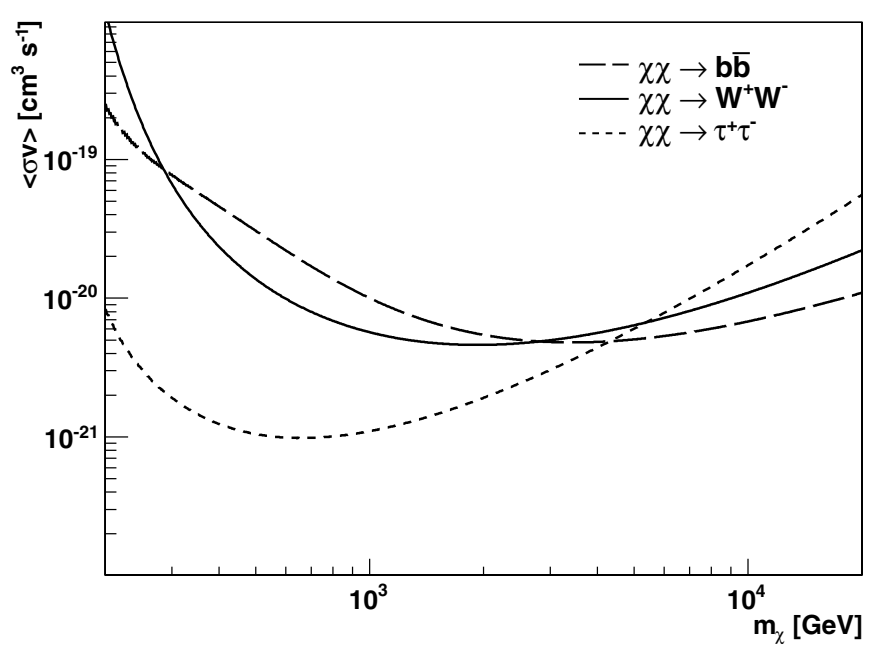

Figure 6. Limits on the DM annihilation cross section $\langle\sigma v\rangle$ from VERITAS observations of the Coma Cluster as a function of the DM particle mass $m_{\chi}$ derived from the VERITAS gamma-ray flux upper limits $(0.2$ aperture $)$ presented in this work.

Table 7

Astrophysical Factors

\begin{tabular}{lcc}
\hline \hline$R(\mathrm{deg})$ & $\langle J\rangle_{\text {signal }}\left(\mathrm{GeV}^{2} \mathrm{~cm}^{-5} \mathrm{sr}\right)$ & $\alpha\langle J\rangle_{\text {bkg }}\left(\mathrm{GeV}^{2} \mathrm{~cm}^{-5} \mathrm{sr}\right)$ \\
\hline 0 & $5.7 \times 10^{16}$ & $1.3 \times 10^{14}$ (negligible $)$ \\
0.2 & $8.1 \times 10^{16}$ & $4.4 \times 10^{14}\left(<0.01\langle J\rangle_{\text {signal }}\right.$, negligible $)$ \\
0.4 & $9.4 \times 10^{16}$ & $1.3 \times 10^{15}\left(\simeq 0.01\langle J\rangle_{\text {signal }}\right.$, negligible $)$ \\
\hline
\end{tabular}

Notes. $\langle J\rangle_{\text {bkg }}$ is the astrophysical factor calculated for the background region (ring method) and is used to estimate the level of gamma-ray contamination from DM annihilation. $\alpha$ is the size ratio of the ON- and OFF-source regions.

contamination from DM annihilation in the background region. As long as the DM contribution to the event number in the background region is negligible, the upper limits derived here directly scale with the astrophysical factor, $\operatorname{UL}(\langle\sigma v\rangle) \propto J^{-1}$. The analysis uses a ring region to estimate the background in an $\mathrm{ON}$ region. We have to compute the expected level of gamma-ray emission from DM annihilation in the ring region in order to check that it is negligible with respect to the level of gamma-ray emission from DM annihilation in the ON region. This is equivalent to compute the astrophysical factor of the $\mathrm{ON}$ - and OFF-source regions since this quantity is related to the rate of DM annihilations.

The resulting exclusion curves in the $\left(\langle\sigma v\rangle, m_{\chi}\right)$ parameter space are shown in Figure 6 for three different DM selfannihilation channels, $\mathrm{W}^{+} \mathrm{W}^{-}, \mathrm{b} \overline{\mathrm{b}}$, and $\tau^{+} \tau^{-}$. Depending on the DM annihilation channel, the limits are on the order of $10^{-20}$ to $10^{-21} \mathrm{~cm}^{3} \mathrm{~s}^{-1}$. The minimum for each exclusion curve and corresponding DM particle mass is listed in Table 8. We stress that these limits are derived with conservative estimates of the astrophysical factor $J$. They do not include any boost to the annihilation rate possibly due to DM substructures populating the Coma halo, which could scale down the present limits by a factor $O(1000)$ in the most optimistic cases (Pinzke et al. 2011; Gao et al. 2012).

We also note that when the size of the integration region is increased, the limits on $\langle\sigma v\rangle$ result from a competition between the gain in the astrophysical factor $\langle J\rangle$ and the integrated background. For integration regions larger than 0.2 in radius, the astrophysical factors no longer compensate for the increased number of background events, and the signal-to-noise ratio deteriorates.
Table 8

Upper Limits on the DM Annihilation Cross Section Times Velocity $\langle\sigma v\rangle$ from VERITAS Observations of the Coma Cluster

\begin{tabular}{lccc}
\hline \hline Channel & $R(\operatorname{deg})$ & $m_{\chi}(\mathrm{GeV})$ & $\langle\sigma v\rangle\left(\mathrm{cm}^{3} \mathrm{~s}^{-1}\right)$ \\
\hline $\mathrm{W}^{+} \mathrm{W}^{-}$ & 0 & 2000 & $1.1 \times 10^{-20}$ \\
& 0.2 & 1900 & $4.3 \times 10^{-21}$ \\
& 0.4 & 1900 & $8.4 \times 10^{-21}$ \\
$b \bar{b}$ & 0 & 3500 & $1.2 \times 10^{-20}$ \\
& 0.2 & 3400 & $4.4 \times 10^{-21}$ \\
\multirow{2}{*}{$\tau^{+} \tau^{-}$} & 0.4 & 3500 & $8.7 \times 10^{-21}$ \\
& 0 & 670 & $2.4 \times 10^{-21}$ \\
& 0.2 & 650 & $9.1 \times 10^{-22}$ \\
& 0.4 & 660 & $1.8 \times 10^{-21}$ \\
\hline
\end{tabular}

Notes. Upper limits are shown for different integration regions and DM particle mass $m_{\chi}$ and are derived from the VERITAS gamma-ray flux upper limits presented in this work.

\section{DISCUSSION AND CONCLUSIONS}

We have reported on the observations of the Coma Cluster of galaxies in VHE gamma rays with VERITAS and complementary observations with the Fermi-LAT. VERITAS observed the Coma Cluster of galaxies for a total of $18.6 \mathrm{hr}$ of high-quality live time between March and May in 2008. No significant excess of gamma rays was detected above an energy threshold of $\sim 220 \mathrm{GeV}$. The Fermi-LAT has observed the Coma Cluster in all-sky survey mode since its launch in 2008 June. We have used all data available from launch to 2012 April for an updated analysis compared to published results (Ackermann et al. 2010). Again, no significant excess of gamma rays was detected. We have used the VERITAS and Fermi-LAT data to calculate flux upper limits at the $99 \%$ confidence level for the cluster core (considered as both a point-like source and a spatially extended emission region) and for three member galaxies. The flux upper limits obtained were then used to constrain properties of the cluster.

We have employed various approaches to constrain the CR population and magnetic field distribution that are complementary in their assumptions and hence well suited to assessment of the underlying Bayesian priors in the models. (1) We used a simplified "isobaric CR model" that is characterized by a constant CR-to-thermal pressure fraction and has a power-law momentum spectrum. While this model is not physically justified a priori, it is simple and widely used in the literature and captures some aspects of more elaborate models such as (2) the simulation-based analytical approach of Pinzke \& Pfrommer (2010). The latter is a "first-principle approach" that predicts the CR distribution spectrally and spatially for a given set of assumptions. It is powerful since it only requires the density profile as input due to the approximate universality of the CR distribution (when neglecting CR diffusion and streaming). Note, however, that inclusion of these CR transport processes may be necessary to explain the radio-halo bimodality. (3) Finally, we used a pragmatic approach that models the CR and magnetic distributions in order to reproduce the observed emission profile of the Coma radio halo. While this approach is also not physically justified, it is powerful because it shows what the "correct" model has to achieve and can point in the direction of the relevant physics.

Within this pragmatic approach, we employ two different methods. First, adopting a high magnetic field everywhere in the cluster $\left(B \gg B_{\mathrm{CMB}}\right.$ ) yields the minimum gamma-ray flux in the hadronic model of radio halos, which we find to be 
a factor of 20 (60) below the most constraining flux upper limits of Fermi-LAT (VERITAS). Second, by matching the radio-emission profile (i.e., fixing the radial $\mathrm{CR}$ profile for a given magnetic field model) and by requiring the pion-decay gamma-ray flux to match the flux upper limits (i.e., fixing the normalization of the CR distribution), we obtain lower limits on the magnetic field distribution under consideration. Our limits for the central magnetic field range from $B_{0}=0.5$ to $1.4 \mu \mathrm{G}$ (for VERITAS flux limits) and from $B_{0}=1.4$ to $5.5 \mu \mathrm{G}$ (for FermiLAT flux limits). Since all (but one) of these lower limits on $B_{0}$ are below the values favored by Faraday RM, $B_{0}=4.7_{-0.8}^{+0.7} \mu \mathrm{G}$ (Bonafede et al. 2010), the hadronic model is a very attractive explanation of the Coma radio halo. The Fermi-LAT upper limits start to rule out the parameter combination of $\alpha_{B} \gtrsim 0.7$ and $\alpha \gtrsim 2.5$ for the hadronic model of the Coma radio halo.

Applying our simplified "isobaric CR model" to the most constraining VERITAS limits, we can constrain the CR-tothermal pressure ratio, $X_{\mathrm{CR}}$, to be below $0.048-0.43$ (for a CR or gamma-ray spectral index, $\alpha$, varying between 2.1 and 2.5). We obtain a constraint of $X_{\mathrm{CR}}<0.1$ for $\alpha=2.3$, the spectral index predicted by simulations at energies around $220 \mathrm{GeV}$. This limit is more constraining by a factor of 1.6 than that of the simulation-based model, which gives $X_{\mathrm{CR}}<0.16$. This difference is due to the concave form of the simulated spectrum, which provides more pressure at $\mathrm{GeV}$ energies in comparison to a pure power-law spectrum of $\alpha=2.3$.

The Fermi-LAT flux limits constrain $X_{\mathrm{CR}}$ to be below $0.012-0.017$ (for $\alpha$ varying between 2.3 and 2.1 ), only weakly depending on the assumed CR spectral index. Assuming $\alpha=$ 2.1 , which is very close to the simulated spectral index for the energy range of $1-3 \mathrm{GeV}$, we obtain a constraint that is identical to that from our simulation-based model within the virial radius of $X_{\mathrm{CR}}<0.017$. That constraint improves to $X_{\mathrm{CR}}<0.012$ for an aperture of 0.4 corresponding to a physical scale of $R \simeq R_{200} / 3 \simeq 660 \mathrm{kpc}$. These upper limits are now starting to constrain the $\mathrm{CR}$ physics in self-consistent cosmological cluster simulations and cap the maximum $\mathrm{CR}$ acceleration efficiency at structure formation shocks to be $<50 \%$. Alternatively, this may argue for non-negligible CR transport processes such as CR streaming and diffusion into the outer cluster regions (Aleksić et al. 2012). These are encouraging results in that we constrain the CR pressure (of a phase that is fully mixed with the ICM) to be at most a small fraction $(<0.017)$ of the overall pressure. As a result, hydrostatic cluster masses and the total Comptonization parameter due to the Sunyaev-Zel'dovich effect suffer at most a very small bias due to CRs.

We have also used the flux upper limits obtained with VERITAS to constrain the thermally averaged product of the total self-annihilation cross section and the relative velocity of DM particles. Modeling the Coma Cluster DM halo with an NFW profile, we derived limits on $\langle\sigma v\rangle$ to be on the order of $10^{-20}$ to $10^{-21} \mathrm{~cm}^{-3} \mathrm{~s}^{-1}$ depending on the chosen aperture. These limits are based on conservative estimates of the astrophysical factor, where a possible boost to the annihilation rate due to DM substructures in the cluster halo has been neglected. Including such a boost could scale down the present limits by a factor $O(1000)$ in the most optimistic cases.

This research is supported by grants from the U.S. Department of Energy Office of Science, the U.S. National Science Foundation, and the Smithsonian Institution, by NSERC in Canada, by Science Foundation Ireland (SFI 10/RFP/AST2748), and by STFC in the U.K. We acknowledge the excellent work of the technical support staff at the Fred Lawrence Whipple Observatory and at the collaborating institutions in the construction and operation of the instrument.

The Fermi-LAT Collaboration acknowledges generous ongoing support from a number of agencies and institutes that have supported both the development and the operation of the LAT, as well as scientific data analysis. These include the National Aeronautics and Space Administration and the Department of Energy in the United States, the Commissariat à l'Energie Atomique and the Centre National de la Recherche Scientifique/Institut National de Physique Nucléaire et de Physique des Particules in France, the Agenzia Spaziale Italiana and the Istituto Nazionale di Fisica Nucleare in Italy, the Ministry of Education, Culture, Sports, Science and Technology (MEXT), High Energy Accelerator Research Organization (KEK), and Japan Aerospace Exploration Agency (JAXA) in Japan, and the K. A. Wallenberg Foundation, the Swedish Research Council, and the Swedish National Space Board in Sweden.

Additional support for science analysis during the operation phase is gratefully acknowledged from the Istituto Nazionale di Astrofisica in Italy and the Centre National d'Études Spatiales in France.

C.P. gratefully acknowledges financial support of the Klaus Tschira Foundation. A.P. acknowledges NSF grant AST-0908480 for support.

\section{REFERENCES}

Abramowski, A., Acero, F., Aharonian, F., et al. 2011, Phys. Rev. Lett., 106, 161301

Acciari, V., Arlen, T., Aune, T., et al. 2010, ApJ, 720, 1174

Ackermann, M., Ajello, M., Allafort, A., et al. 2010, ApJ, 717, L71

Aharonian, F., Akhperjanian, A., Barrio, J., et al. 2001, A\&A, 370, 112

Aharonian, F. A., Akhperjanian, A. G., Anton, G., et al. 2009a, A\&A, 503, 817 Aharonian, F. A., Akhperjanian, A. G., Anton, G., et al. 2009b, A\&A, 502, 437 Aharonian, F. A., Akhperjanian, A. G., Bazer-Bachi, A. R., et al. 2006, Phys. Rev. Lett., 97, 221102

Aharonian, F. A., Hofmann, W., Konopelko, A. K., \& Völk, H. J. 1997, Astropart. Phys., 6, 343

Ajello, M., Rebusco, P., Cappelluti, N., et al. 2009, ApJ, 690, 367

Ajello, M., Rebusco, P., Cappelluti, N., et al. 2010, ApJ, 725, 1688

Aleksić, J., Alvarez, E. A., Antonelli, L. A., et al. 2012, A\&A, 541, A99

Aleksić, J., Antonelli, L. A., Antoranz, P., et al. 2010, ApJ, 710, 634

Aliu, E., Anderhub, H., Antonelli, L. A., et al. 2009, ApJ, 697, 1299

Battaglia, N., Pfrommer, C., Sievers, J. L., Bond, J. R., \& Enßlin, T. A. 2009, MNRAS, 393, 1073

Berezinsky, V. S., Blasi, P., \& Ptuskin, V. S. 1997, ApJ, 487, 529

Bergström, L., \& Hooper, D. 2006, Phys. Rev. D, 73, 063510

Bonafede, A., Dolag, K., Stasyszyn, F., Murante, G., \& Borgani, S. 2011, MNRAS, 418, 2234

Bonafede, A., Feretti, L., Murgia, M., et al. 2010, A\&A, 513, A30

Briel, U. G., Henry, J. P., \& Böhringer, H. 1992, A\&A, 259, L31

Briel, U. G., Henry, J. P., Lumb, D. H., et al. 2001, A\&A, 365, L60

Brown, S., \& Rudnick, L. 2011, MNRAS, 412, 2

Brunetti, G., \& Lazarian, A. 2011, MNRAS, 410, 127

Bullock, J. S., Kolatt, T. S., Sigad, Y., et al. 2001, MNRAS, 321, 559

Cassano, R., Ettori, S., Giacintucci, S., et al. 2010, ApJ, 721, L82

Churazov, E., Forman, W., Vikhlinin, A., et al. 2008, MNRAS, 388, 1062

Churazov, E., Tremaine, S., Forman, W., et al. 2010, MNRAS, 404, 1165

Cogan, P. for the VERITAS Collaboration 2008, in Proc. 30th Int. Cosmic Ray

Conf., Vol. 3, ed. G. M.-T. R. Caballero \& J. C. D'Olivo et al. (Mexico City:

Universidad Nacional Autónoma de México), 1385

Colafrancesco, S., \& Blasi, P. 1998, Astropart. Phys., 9, 227

Cuesta, A. J., Jeltema, T. E., Zandanel, F., et al. 2011, ApJ, 726, L6

Daniel, M. K. for the VERITAS Collaboration 2008, in Proc. 30th Int. Cosmic

Ray Conf., Vol. 3, ed. G. M.-T. R. Caballero \& J. C. D'Olivo et al. (Mexico

City: Universidad Nacional Autónoma de México), 1325

Deiss, B. M., Reich, W., Lesch, H., \& Wielebinski, R. 1997, A\&A, 321, 55

Dennison, B. 1980, ApJ, 239, L93

Diaferio, A., Schindler, S., \& Dolag, K. 2008, Space Sci. Rev., 134, 7

Dolag, K., \& Enßlin, T. A. 2000, A\&A, 362, 151 
Eckert, D., Neronov, A., Courvoisier, T. J.-L., \& Produit, N. 2007, A\&A, 470, 835

Enßlin, T. A. 2002, A\&A, 396, L17

Enßlin, T. A., Biermann, P. L., Klein, U., \& Kohle, S. 1998, A\&A, 332, 395

Enßlin, T. A., Pfrommer, C., Miniati, F., \& Subramanian, K. 2011, A\&A, 527, A99

Enßlin, T. A., Pfrommer, C., Springel, V., \& Jubelgas, M. 2007, A\&A, 473, 41 Evans, N. W., Ferrer, F., \& Sarkar, S. 2004, Phys. Rev. D, 69, 123501

Fomin, V., Stepanian, A. A., Lamb, R. C., et al. 1994, Astropart. Phys., 2, 137

Fusco-Femiano, R., Orlandini, M., Brunetti, G., et al. 2004, ApJ, 602, L73

Gao, L., Navarro, J. F., Frenk, C. S., et al. 2012, arXiv:1201.1940

Gavazzi, R., Adami, C., Durret, F., et al. 2009, A\&A, 498, L33

Giovannini, G., Feretti, L., Venturi, T., Kim, K.-T., \& Kronberg, P. P. 1993, ApJ, 406, 399

Govoni, F., Enßlin, T. A., Feretti, L., \& Giovannini, G. 2001, A\&A, 369, 441

Helder, E. A., Vink, J., Bassa, C. G., et al. 2009, Science, 325, 719

Hillas, A. M. 1985, Proc. 19th Int. Cosmic Ray Conf. (Washington, DC: NASA), 445

Holder, J., Atkins, R. W., Badran, H. M., et al. 2006, Astropart. Phys., 25, 391

Jubelgas, M., Springel, V., Enßlin, T. A., \& Pfrommer, C. 2008, A\&A, 481, 33

Kang, H., \& Jones, T. W. 2005, ApJ, 620, 44

Kosack, K., Badran, H. M., Bond, I. H., et al. 2004, ApJ, 608, L97

Krawczynski, H., Carter-Lewis, D. A., Duke, C., et al. 2006, Astropart. Phys., 25,380

Kubo, J. M., Stebbins, A., Annis, J., et al. 2008, ApJ, 671, 1466

Li, T., \& Ma, Y. 1983, ApJ, 272, 317

Mahdavi, A., Hoekstra, H., Babul, A., \& Henry, J. P. 2008, MNRAS, 384, 1567

Maier, G., \& Knapp, J. 2007, Astropart. Phys., 28, 78

Mattox, J. R., Bertsch, D. L., Chiang, J., et al. 1996, ApJ, 461, 396

Miniati, F. 2003, MNRAS, 342, 1009

Miniati, F., Ryu, D., Kang, H., \& Jones, T. W. 2001, ApJ, 559, 59

Navarro, J. F., Frenk, C. S., \& White, S. D. M. 1997, ApJ, 490, 493

Neumann, D. M., Lumb, D. H., Pratt, G. W., \& Briel, U. G. 2003, A\&A, 400, 811

Nolan, P. L., Abdo, A. A., Ackermann, M., et al. 2012, ApJS, 199, 31

Perkins, J. 2008, in AIP Conf. Ser. 1085, High-energy Gamma-ray Astronomy: Proc. 4th International Meeting on High Energy Gamma-ray Astronomy, ed. F. A. Aharonian, W. Hofmann, \& F. Rieger (Melville, NY: AIP), 569
Perkins, J. S., Badran, H. M., Blaylock, G., et al. 2006, ApJ, 644, 148 Petrosian, V. 2001, ApJ, 557, 560

Pfrommer, C. 2008, MNRAS, 385, 1242

Pfrommer, C., \& Enßlin, T. A. 2004a, A\&A, 413, 17

Pfrommer, C., \& Enßlin, T. A. 2004b, MNRAS, 352, 76

Pfrommer, C., Enßlin, T. A., \& Springel, V. 2008, MNRAS, 385, 1211

Pfrommer, C., Enßlin, T. A., Springel, V., Jubelgas, M., \& Dolag, K. 2007, MNRAS, 378, 385

Pfrommer, C., Springel, V., Enßlin, T. A., \& Jubelgas, M. 2006, MNRAS, 367, 113

Pinzke, A., \& Pfrommer, C. 2010, MNRAS, 409, 449

Pinzke, A., Pfrommer, C., \& Bergström, L. 2009, Phys. Rev. Lett., 103, 181302

Pinzke, A., Pfrommer, C., \& Bergstrom, L. 2011, Phys. Rev. D, 84, 123509

Pohl, M. 1994, A\&A, 287, 453

Reimer, O., Pohl, M., Sreekumar, P., \& Mattox, J. R. 2003, ApJ, 588, 155

Rephaeli, M., \& Gruber, D. 2002, ApJ, 597, 587

Rolke, W. A., Lopez, A. M., \& Conrad, J. 2005, Nucl. Intrum. Methods Phys. Res. A, 551, 493

Ryu, D., Kang, H., Hallmann, H., \& Jones, T. W. 2003, ApJ, 593, 599

Sarazin, C. L., \& Lieu, R. 1983, ApJ, 270, 422

Schlickeiser, R., Sievers, A., \& Thiemann, H. 1987, A\&A, 182, 21

Schuecker, P., Finoguenov, A., Miniati, F., Böhringer, H., \& Briel, U. G. 2004, A\&A, 426, 387

Skillman, S. W., Hallman, E. J., O’Shea, B. W., et al. 2011, ApJ, 735, 96

Smith, H. J. 1998, ApJ, 494, L177

Sreekumar, P., Bertsch, D. L., Dingus, B. L., et al. 1996, ApJ, 464, 628

Strigari, L. E., Koushiappas, S. M., Bullock, J. S., \& Kaplinghat, M. 2007, Phys. Rev. D, 75, 083526

Struble, M. F., \& Rood, H. J. 2003, ApJ, 593, 599

Thierbach, M., Klein, U., \& Wielebinski, R. 2003, A\&A, 397, 53

Tribble, P. C. 1993, MNRAS, 263, 31

Vikhlinin, A., Markevitch, M., Murray, S. S., et al. 2005, ApJ, 628, 655

Voges, W., Aschenbach, B., Boller, Th., et al. 1999, A\&A, 349, 389

Voit, G. M. 2005, Rev. Mod. Phys., 77, 207

Völk, H., Aharonian, F. A., \& Breitschwerdt, D. 1996, Space Sci. Rev., 75, 279

Völk, H. J. 1989, A\&A, 218, 67

Weekes, T. C., Badran, H., Biller, S. D., et al. 2002, Astropart. Phys., 17, 221

Wik, D. R., Sarazin, C. L., Finoguenov, A., et al. 2009, ApJ, 696, 1700 